\title{
Embedded polar spaces revisited
}

\author{
Antonio Pasini
}

\begin{abstract}
Pseudo-quadratic forms have been introduced by Tits [10, Chapter 8] in view of the classification of polar spaces. A slightly different notion is proposed by Tits and Weiss [11, Chapter 11]. In this paper we propose a generalization of the definition of [10], inspired by [11]. With its help we will be able to clarify a few points in the classification of embedded polar spaces. We recall that, according to [10], given a division ring $K$ and an admissible pair $(\sigma, \varepsilon)$ in it, the codomain of a $(\sigma, \varepsilon)$-quadratic form is the group $\bar{K}:=K / K_{\sigma, \varepsilon}$, where $K_{\sigma, \varepsilon}:=\left\{t-t^{\sigma} \varepsilon\right\}_{t \in K}$. Our generalization amounts to replace $\bar{K}$ with a quotient $\bar{K} / \bar{R}$ for a subgroup $\bar{R}$ of $\bar{K}$ such that $\lambda^{\sigma} \bar{R} \lambda=\bar{R}$ for any $\lambda \in K$. We call generalized pseudo-quadratic forms (also generalized $(\sigma, \varepsilon)$-quadratic forms) the forms defined in this more general way, keeping the words pseudo-quadratic form and $(\sigma, \varepsilon)$-quadratic form for those defined as in [10]. Generalized pseudo-quadratic forms behave just like pseudo-quadratic forms. In particular, every non-trivial generalized pseudo-quadratic form admits a unique sesquilinearization, characterized by the same property as the sesquilinearization of a pseudo-quadratic form. Moreover, if $q: V \rightarrow \bar{K} / \bar{R}$ is a non-trivial generalized pseudo-quadratic form and $f: V \times V \rightarrow K$ is its sesquilinearization, the points and the lines of $\mathrm{PG}(V)$ where $q$ vanishes form a subspace $S_{q}$ of the polar space $S_{f}$ associated to $f$. In this paper, after a discussion of quotients and covers of generalized pseudo-quadratic forms, we shall prove the following, which sharpens a celebretated theorem of Buekenhout and Lefèvre [3]. Let $e: S \rightarrow \mathrm{PG}(V)$ be a projective embedding of a non-degenerate polar space $S$ of rank at least 2 ; then $e(S)$ is either the polar space $S_{q}$ associated to a generalized pseudo-quadratic form $q$ or the polar space $S_{f}$ associated to an alternating form $f$. By exploiting this theorem we also obtain an elementary proof of the following well known fact: an embedding $e$ as above is dominant if and only if either $e(S)=S_{q}$ for a pseudo-quadratic form $q$ or $\operatorname{char}(K) \neq 2$ and $e(S)=S_{f}$ for an alternating form $f$.
\end{abstract}

Keywords : polar spaces, embeddings

MSC 2010: 51A50, 51A45, 51E12, 51E24. 


\section{Introduction}

\subsection{Polar spaces and their embeddings}

We refer to Tits [10, Chapters 7 and 8] and Buekenhout and Cohen [2, Chapters 7-10] for the theory of polar spaces and their projective embeddings, but we warn the reader that there are some differences between the setting chosen by Tits [10] and the approach of Buekenhout and Cohen [2]. To begin with, the definition of polar space adopted in [2] (which is the same as in Buekenhout and Shult [4]) is more general than that of Tits [10]: a polar space as defined by Tits [10, Chapter 7] is a non-degenerate polar space of finite rank in the sense of [2]. In this paper we shall stick to the definition of [2], according to which a polar space is a point-line geometry $S=(P, L)$ such that for every point $p \in P$ and every line $l \in L$, the point $p$ is collinear with either all or just one of the points of $l$. The notion of projective embedding used in [10, Chapter 8] also looks more restrictive than that of [2], although those two notions are in fact equivalent, as we will see in a few lines. According to [2], an embedding of a polar space $S=(P, L)$ is an injective mapping $e$ from the point-set $P$ of $S$ to the set of points of the projective geometry $\mathrm{PG}(V)$ of a vector space $V$, such that $e$ maps every line of $S$ surjectively onto a line of $\mathrm{PG}(V)$ and $e(P)$ spans $\mathrm{PG}(V)$ (compare our definition of embeddings in Subsection 1.3.3), while Tits [10] also assumes the following:

$(*)$ The image $e(S)=(e(P), e(L))$ of $S$ by $e$ is a subspace of the polar space $S_{f}$ associated to a (possibly degenerate) reflexive sesquilinear form $f$ : $V \times V \rightarrow K$.

Needless to say, $K$ is the underlying division ring of $V$. As for the definition of subspaces, we refer the reader to Subsection 1.3.1 of this paper. Note that the hypothesis that $e(S)$ is a subspace of $S_{f}$ uniquely determines $f$ modulo proportionality (Tits [10, Chapter 8], Buekenhout and Cohen [2, Chapter 9]).

However, as we said above, these two definitions of embedding are practically the same. Indeed:

Theorem 1.1 (Buekenhout and Cohen [2, Chapter 9]). Let e be a projective embedding of a polar space $S$, in the sense of [2]. Suppose that $S$ is non-degenerate of rank at least 2 . Then $(*)$ holds for $e$.

To my knowledge, the earliest version of Theorem 1.1 that has appeared in the literature is due to Dienst [6], who completed the work formerly done by Buekenhout and Lefèvre [3] on embeddings of finite polar spaces of rank 2. 
Dienst still sticks to the rank 2 case in [6], but his arguments also work for higher rank polar spaces, modulo a few obvious adjustments.

In view of the next theorem we need a few definitions. We state them now for embeddings of polar spaces but we shall turn back to them in Subsection 1.3.3, in a more general context. Referring to Subsection 1.3.3 for quotients and covers of embeddings, we say that a projective embedding of a polar space $S$ is dominant if it is not a proper quotient of any other embedding of $S$. In other words, it is not properly covered by any other embedding. It is well known that every embedding $e$ is covered by a dominant embedding, uniquely determined by $e$ up to isomorphism and called the hull of $e$ (see Section 1.3.3). An embedding $e$ of $S$ is initial if all projective embeddings of $S$ are quotients of $e$. Clearly the initial embedding, if it exists, is unique up to isomorphism. It is in fact the unique dominant embedding of $S$. In other words, $S$ admits the initial embedding if and only if all embeddings of $S$ admit the same hull.

We refer to Tits $[10,8.2]$ (also Section 2 of the present paper) for the definition of pseudo-quadratic forms.

Theorem 1.2 (Tits $[10,8.6])$. Let $S$ be a non-degenerate polar space of rank at least 2 and let $e: S \rightarrow \mathrm{PG}(V)$ be a projective embedding of $S$, with $e(S)$ a subspace of $S_{f}$ as in $(*)$. Then $e$ is dominant if and only if one of the following holds:

(1) The form $f$ is alternating, the underlying field of $V$ has characteristic other than 2 and $e(S)=S_{f}$.

(2) The image $e(S)$ of $S$ is the polar space $S_{q}$ associated to a non-singular pseudo-quadratic form $q$ such that $f$ is the sesquilinearization of $q$.

Moreover, if e is dominant then it is also initial, except for two exceptional cases where $S$ has rank 2.

The two exceptional cases mentioned above will be described later in this paper (Section 6, Theorem 6.4). We now turn to the most important theorem of the theory of polar spaces.

Theorem 1.3 (Tits [10]). Let $S$ be a non-degenerate polar space of rank at least 3. Suppose that the planes of $S$ are desarguesian. Moreover, when $S$ has rank 3 and every line of $S$ belongs to exactly two planes, suppose also that the planes of $S$ are Pappian. Then $S$ admits a projective embedding.

Tits proves Theorem 1.3 in Chapter 8 of [10]. A different proof, inspired by the work of Veldkamp [12], is offered by Buekenhout and Cohen in [2, Chapter 10]. Tits's proof is rather algebraic in flavour. He constructs an embedding of $S$ by a free construction where vector spaces associated to the singular subspaces of $S$ containing a given point of $S$ are amalgamated so that to obtain a 
vector space $\bar{V}$ which, extended by adding two copies of the underlying division ring $K$ of $S$, yields a vector space $\widetilde{V}=\bar{V} \oplus V(2, K)$ which hosts an embedding $\tilde{e}$ of $S$. The embedding $\tilde{e}$ constructed in that way is initial. Explicitly, let $\tilde{f}$ be the reflexive sesquilinear form on $\widetilde{V}$ such that $\tilde{e}$ is a subspace of $S_{\tilde{f}}($ see $(*))$. If $\tilde{e}(S)=S_{\tilde{f}}$ then $\tilde{f}$ is non-degenerate and $\tilde{e}$ is the unique projective embedding of $S$. Otherwise, $\tilde{f}$ is the sesquilinearization of a non-singular pseudo-quadratic form $\tilde{q}$, we have $\tilde{e}(S)=S_{\tilde{q}}$ and all projective embeddings of $S$ arise as quotients of $\tilde{e}$ over a subspace of the $\operatorname{radical} \operatorname{Rad}(\tilde{f})$ of $\tilde{f}$. Thus we also have a complete classification of projective embeddings of non-degenerate polar spaces of rank at least 3.

The proof by Buekenhout and Cohen is completely geometric. Following the original approach by Veldkamp [12], they prove that the family of hyperplanes of $S=(P, L)$ (see Subsection 1.3.1 for the definition of hyperplanes) forms a projective space, say it $\mathcal{V}(S)$, called the Veldkamp space of $S$. The hyperplanes of $S$ are the points of $\mathcal{V}(S)$ while the lines of $\mathcal{V}(S)$ are families of hyperplanes consisting of all hyperplanes of $S$ containing the intersection of two given hyperplanes. As $S$ is non-degenerate by assumption, for every point $p \in P$ the set of points of $S$ collinear with $p$ is a hyperplane of $S$, hence a point of $\mathcal{V}(S)$, usually denoted by the symbol $p^{\perp}$. Let $\hat{e}$ be the mapping from the point-set of $S$ to the set of points of $\mathcal{V}(S)$ defined by setting $\hat{e}(p)=p^{\perp}$ for every $p \in P$. Then $\hat{e}$ is an embedding of $S$ in the subspace $\widehat{V}$ of $\mathcal{V}(S)$ spanned by $\hat{e}(P)$. We call $\hat{e}$ the Veldkamp embedding of $S$.

In a sense, the Veldkamp embedding $\hat{e}$ is the counterpart of the initial embedding $\tilde{e}$ constructed by Tits. Indeed, while $\tilde{e}$ covers all embeddings of $S$, the Veldkamp embedding is covered by all of them. In short, $\hat{e}$ is terminal. We obtain it from $\tilde{e}$ by factorizing $\tilde{e}$ over $\operatorname{Rad}(\tilde{f})$.

In order to classify the embeddings of $S$ we should now describe all covers of $\hat{e}$. In particular, we must show how to recover $\tilde{e}$ from $\hat{e}$, but possibly without exploiting Tits's construction of $\tilde{e}$. However, if we want to do so, Theorem 1.1 is the only tool we have at hand. According to that theorem, if $e$ is an embedding of $S$ then $e(S)$ is a subspace of $S_{f}$ for a suitable reflexive sesquilinear form $f$, but it can happen that $e(S)$ is a proper subspace of $S_{f}$ as well as a proper overspace of $S_{q}$ for every pseudo-quadratic form $q$ admitting $f$ as the sequilinearization. As a consequence, if $\hat{f}$ is the $(\sigma, \varepsilon)$-sesquilinear form on $\widehat{V}$ such that $\hat{e}(S)$ is a subspace of $S_{\hat{f}}$ and $\tilde{e}$ is the initial embedding of $S$, we can only claim that $\tilde{e}(S)=S_{\tilde{q}}$ for a suitable $(\sigma, \varepsilon)$-quadratic form $\tilde{q}$ defined on a suitable subspace $\tilde{V}$ of $\widehat{V} \oplus K^{\sigma, \varepsilon} / K_{\sigma, \varepsilon}$ (compare Buekenhout and Cohen [2, Theorem 10.12.5]). This is admittedly a bit vague, even if in many particular cases we can easily explain which subspace $\widetilde{V}$ actually is. It would be nice to have a precise description of $\widetilde{V}$, valid in general. 


\subsection{Purpose and main results of this paper}

The purpose of this paper is to overcome the difficulties discussed in the previous paragraph. We will succeed by using a definition of pseudo-quadratic form more general than that of [10], inspired by Tits and Weiss [11, Chapter 11]. We recall that, according to Tits $[10,8.2]$, given a division ring $K$, an anti-automorphism $\sigma$ of $K$ and an element $\varepsilon \in K^{*}$ such that $\varepsilon^{1+\sigma}=1$ and $t^{\sigma^{2}}=\varepsilon t \varepsilon^{-1}$ for every $t \in K$, the codomain of a $(\sigma, \varepsilon)$-quadratic form is the group $\bar{K}:=K / K_{\sigma, \varepsilon}$, where $K_{\sigma, \varepsilon}:=\left\{t-t^{\sigma} \varepsilon\right\}_{t \in K}$. In our generalization we keep the definition of $[10,8.2]$ but we replace $\bar{K}$ with a quotient $\bar{K} / \bar{R}$, where $\bar{R}$ is any subgroup of $\bar{K}$ such that $\lambda^{\sigma} \bar{R} \lambda=\bar{R}$ for every $\lambda \in K$. In order to avoid any confusion, we call the forms defined in this more general way generalized $(\sigma, \varepsilon)$-quadratic forms (also generalized pseudo-quadratic forms, with no mention of the pair $(\sigma, \varepsilon)$ when possible), keeping the words $(\sigma, \varepsilon)$-quadratic form and pseudo-quadratic form for pseudo-quadratic forms defined as in [10, 8.2].

As we shall show in Section 3, most of the properties of pseudo-quadratic forms also hold for generalized pseudo-quadratic forms. In particular, every non-trivial generalized pseudo-quadratic form admits a unique sesquilinearization, characterized by the same property as the sesquilinearization of a pseudoquadratic form. Moreover, if $q: V \rightarrow \bar{K} / \bar{R}$ is a non-trivial generalized pseudoquadratic form and $f: V \times V \rightarrow K$ is its sesquilinearization, then the points and the lines of $\mathrm{PG}(V)$ where $q$ vanishes form a subspace $S_{q}$ of $S_{f}$. In Section 5 (Theorems 5.5 and 5.8) we shall obtain the following improvement of Theorem 1.1:

Theorem 1.4. Let e $: S \rightarrow \mathrm{PG}(V)$ be a projective embedding of a non-degenerate polar space $S$ of rank at least 2 . Then $e(S)$ is either the polar space $S_{q}$ associated to a non-trivial generalized pseudo-quadratic form $q$ or the polar space $S_{f}$ associated to a non-degenerate alternating form $f$.

As said before, the hull of an embedding $e$ is the unique dominant embedding that covers $e$. With $e$ and $S$ as in Theorem 1.4, the hull of $e$ is the initial embedding of $S$, with the only exception of the two cases of rank 2 mentioned in the last claim of Theorem 1.2.

Let $e(S)=S_{f}$ for an alternating form $f$ and let $\tilde{e}$ be the hull of $e$. It is well known that in this case either $\tilde{e}=e$ (when $\operatorname{char}(K) \neq 2$ ) or $\operatorname{char}(K)=2$ and $\tilde{e}(S)=S_{\tilde{q}}$ for a non-singular quadratic form $\tilde{q}: \widetilde{V} \rightarrow K$, where $\tilde{V}=V \oplus K$, the field $K$ being regarded as a vector space over itself with scalar multiplication $\circ: K \times K \rightarrow K$ defined as follows: $t \circ \lambda=t \lambda^{2}$ for every vector $t \in K$ and every scalar $\lambda \in K$.

On the other hand, let $e(S)=S_{q}$ for a generalized pseudo-quadratic form $q: V \rightarrow \bar{K} / \bar{R}$. Let $\circ: \bar{R} \times K \rightarrow K$ be defined as follows: $r \circ \lambda=\lambda^{\sigma} r \lambda$ for every 
$r \in \bar{R}$ and every scalar $\lambda \in K$. We will prove in Section 3 that the group $\bar{R}$ equipped with $\circ$ as the scalar multiplication is a $K$-vector space. (This amounts to say that $\bar{R} \subseteq K^{\sigma, \varepsilon} / K_{\sigma, \varepsilon}$.) Hence we can form a direct sum of $K$-vector spaces $\widetilde{V}=V \oplus \bar{R}$ and, if $f$ is the sesquilinearization of $q$, we can define a reflexive sesquiliner form $\tilde{f}: \widetilde{V} \times \widetilde{V} \rightarrow K$ by declaring that $\bar{R} \subseteq \operatorname{Rad}(\tilde{f})$ and $\tilde{f}$ induces $f$ on $V \times V$. As we shall prove in Section 4, a pseudo-quadratic form $\tilde{q}: \widetilde{V} \rightarrow \bar{K}$ can be defined admitting $\tilde{f}$ as its sesquilinearization and such that the projection $\pi: \widetilde{V} \rightarrow \widetilde{V} / \bar{R}=V$ induces an isomorphism $\pi_{S}$ from $S_{\tilde{q}}$ to $S_{q}$. So, the mapping $\tilde{e}:=\pi_{S}^{-1} \cdot e$ is a projective embedding of $S$ and $\pi$ is a morphism from $\tilde{e}$ to $e$. Moreover, $\tilde{e}$ is dominant by Theorem 1.2, since $\tilde{e}(S)=S_{\tilde{q}}$ and $\tilde{q}$ is pseudo-quadratic. Therefore:

Theorem 1.5. The hull of $e$ is the embedding ẽ defined as above.

\subsection{Subspaces and embeddings of point-line geometries}

So far we have freely mentioned embeddings and subspaces. It is time to fix these notions in a proper way.

Throughout this subsection $G=(P, L)$ is a point-line geometry, with $P$ and $L$ as the point-set and the line-set respectively. We regard lines as subsets of $P$ and we assume that no two distinct lines meet in more than one point and every line has at least two points. The collinearity graph of $G$ is the graph with $P$ as the vertex-set where two points $a, b \in P$ are declared to be adjacent when they are joined by a line of $G$. The geometry $G$ is said to be connected if its collinearity graph is connected.

Given two point-line geometries $G=(P, L)$ and $G^{\prime}=\left(P^{\prime}, L^{\prime}\right)$, an isomorphism from $G$ to $G^{\prime}$ is a bijective mapping $e: P \rightarrow P^{\prime}$ such that $\{e(l)\}_{l \in L}=L^{\prime}$, where for a line $l \in L$ we put $e(l):=\{e(p)\}_{p \in l}$.

\subsubsection{Subgeometries and subspaces}

A point-line geometry $G^{\prime}=\left(P^{\prime}, L^{\prime}\right)$ is a subgeometry of $G=(P, L)$ if $P^{\prime} \subseteq P$ and for every line $l^{\prime} \in L^{\prime}$ there exists a (necessarily unique) line $l \in L$ such that $l^{\prime}=l \cap P^{\prime}$. If every line of $G^{\prime}$ is also a line of $G$ then $G^{\prime}$ is called a full subgeometry of $G$. On the other hand, if $L^{\prime}=\left\{l \cap P^{\prime}|l \in L|, l \cap P^{\prime} \mid \geq 2\right\}$ then $G^{\prime}$ is called the subgeometry induced by $G$ on $P^{\prime}$.

A subset $P^{\prime} \subseteq P$ is called a subspace of $G$ if every line of $G$ either is contained in $P^{\prime}$ or meets $P^{\prime}$ in at most one point. We say that a geometry $G^{\prime}=\left(P^{\prime}, L^{\prime}\right)$ is a subspace of $(G, L)$ if $P^{\prime}$ is a subspace of $G$ in the previous sense and $G^{\prime}$ is the 
subgeometry induced by $G$ on $P^{\prime}$. Clearly, subspaces in the latter sense are full subgeometries.

We have mentioned hyperplanes in Subsection 1.1. A hyperplane of a pointline geometry $G=(P, L)$ is a proper subspace $H \subset P$ such that every line of $G$ either meets $H$ in a single point or is fully contained in $H$.

\subsubsection{Notation for vector spaces and projective spaces}

In view of the next subsection, it is convenient to fix some notation for vector spaces and related projective spaces. Given a vector space $V$, we denote by $\mathrm{PG}(V)$ the projective space of 1- and 2-dimensional vector subspaces of $V$. For a vector $v \in V-\{0\}$, we denote by $[v]$ the projective point of $\mathrm{PG}(V)$ represented by $v$. If $X$ is a subspace of $V$ we put $[X]=\{[x]\}_{x \in X-\{0\}}$, namely $[X]$ is the subspace of $\mathrm{PG}(V)$ corresponding to $X$. Given a semilinear mapping $f: V \rightarrow$ $V^{\prime}$, let $\operatorname{Ker}(f):=f^{-1}(0)$ be the kernel of $f$. We denote by $\mathrm{PG}(f)$ the mapping induced by $f$ from $\mathrm{PG}(V)-[\operatorname{Ker}(f)]$ to $\mathrm{PG}\left(V^{\prime}\right)$.

\subsubsection{Projective embeddings}

Let $G=(P, L)$ be a connected point-line geometry. A projective embedding of $G$ (also called just embedding for short) is an isomorphism $e$ from $G$ to a full subgeometry $e(G)=(e(P), e(L))$ of the projective space $\mathrm{PG}(V)$ of a vector space $V$, such that $e(P)$ spans $\mathrm{PG}(V)$. We write $e: G \rightarrow \mathrm{PG}(V)$ to mean that $e$ is a projective embedding of $G$ in $\mathrm{PG}(V)$. If $K$ is the underlying division ring of $V$ then we say that $e$ is defined over $K$, also that $e$ is a $K$-embedding. If all projective embeddings of $G$ are defined over the same division ring $K$ then we say that $G$ is defined over $K$ and we call $K$ the underlying division ring of $G$.

Given two $K$-embeddings $e: G \rightarrow \mathrm{PG}(V)$ and $e^{\prime} \rightarrow \mathrm{PG}\left(V^{\prime}\right)$, a morphism $f: e \rightarrow e^{\prime}$ is a semilinear mapping $f: V \rightarrow V^{\prime}$ such that $\mathrm{PG}(f) \cdot e=e^{\prime}$. As $e^{\prime}(P)$ spans $\mathrm{PG}\left(V^{\prime}\right)$, the mapping $f$ is surjective. If $f$ is bijective then $f$ is said to be an isomorphism from $e$ to $e^{\prime}$. If a morphism $f: e \rightarrow e^{\prime}$ exists then we say that $e^{\prime}$ is a homomorphic image of $e$ (also that $e$ covers $e^{\prime}$ ) and we write $e \geq e^{\prime}$. If moreover $f$ is bijective then we write $e \cong e^{\prime}$ and we say that $e$ and $e^{\prime}$ are isomorphic, otherwise we call $f$ a proper morphism and we write $e>e^{\prime}$. Note that, as $G$ is connected by assumption, if $e \geq e^{\prime}$ then the morphism $f: e \rightarrow e^{\prime}$ is unique up to isomorphism.

Let $U$ be a subspace of $V$ such that $e(P) \cap[U]=\emptyset$ and $l \cap[U]=\emptyset$ for any line $l$ of $\mathrm{PG}(V)$ such that $|l \cap e(P)| \geq 2$. Let $\pi_{U}$ be the projection of $V$ onto $V / U$. Then the mapping $e_{U}:=\mathrm{PG}\left(\pi_{U}\right) \cdot e$ is an embedding of $G$ in $\mathrm{PG}(V / U)$ and $\pi_{U}$ is a morphism from $e$ to $e_{U}$. We say that $U$ defines a quotient of $e$ and we call 
$e_{U}$ the quotient of $e$ over $U$. If $f: e \rightarrow e^{\prime}$ is a morphism then $\operatorname{Ker}(f)$ defines a quotient of $e$ and $e^{\prime} \cong e_{U}$. By a little abuse, we say that $e^{\prime}$ is a quotient of $e$, thus taking the word 'quotient' as a synonym of 'homomorphic image'.

Following Tits [10, Chapter 8] we say that a projective embedding of $G$ is dominant if it cannot be obtained as a proper quotient from any other projective embedding of $G$. If all $K$-embeddings of $G$ are quotients of a given $K$-embedding $e$ then we say that $e$ is $K$-initial. If moreover $G$ is defined over $K$ then we say that $e$ is absolutely initial, also just initial for short. Thus, when we say that $G$ admits the (absolutely) initial embedding, without mentioning any division ring explicitly, we understand that $G$ is defined over some division ring.

Clearly, the $(K$-)initial embedding, if it exists, is uniquely determined up to isomorphism. It is the unique dominant ( $K$-)embedding of $G$.

Finally, every embedding $e$ of $G$ admits a hull $\tilde{e}$, uniquely determined up to isomorphism by the following property: $\tilde{e} \geq e^{\prime}$ for every embedding $e^{\prime}$ of $G$ such that $e^{\prime} \geq e$. We refer the reader to Ronan [9] for an explicit construction of $\tilde{e}$. Clearly, the hull $\tilde{e}$ of $e$ is dominant. Up to isomorphism, it is the unique dominant embedding in the class of the embeddings that cover $e$. So, if $G$ admits the $K$-initial embedding and $e$ is defined over $K$, then $\tilde{e}$ is also $K$-initial.

The terminology adopted in the previous definitions is essentially the same as in Tits [10], but different terminologies are also used in the literature. For instance, dominant and initial embeddings are often called relatively universal and absolutely universal respectively (compare Kasikova and Shult [8]).

Added in Proof. When this paper was already at the final step of the editing process by the journal, I have learned from Tom De Medts that generalized pseudo-quadratic forms are considered also by A. J. Hahn and O. T. O'Meara in their book The Classical Groups and K-Theory [7]. Indeed, at Section 5.1C of that book, Hahn and O'Meara introduce $\Lambda$-quadratic forms, which are just the same as generalized pseudo-quadratic forms as defined in this paper. Moreover, arbitrary rings with unit are considered by Hahn and O'Meara instead of division rings. It is also worth mentioning that Hahn and O'Meara give Bak credit for having been the first to introduce this notion in full generality [1].

\section{Preliminaries}

In this section we fix some notation and recall a few basics on sesquilinear and pseudo-quadratic forms, taken from Tits [10, Chapter 8] and Buekenhout and Cohen [2, Chapters 7 and 10]. This recapitulation will be exploited in 
Section 3, where generalized pseudo-quadratic forms will be discussed. Indeed many properties of pseudo-quadratic forms hold for generalized pseudoquadratic forms as well, even with the same proofs but for a few obvious modifications. We could urge the reader to look for those proof in the literature and check that they remain valid in the more general setting of Section 3, but we have preferred to take a more friendly attitude. Thus, a few of those proofs will also be sketched in this section, chosen among those that are presumably less well known to non-specialists.

\subsection{Admissible pairs}

Throughout this paper $K$ is a possibly non-commutative division ring, $\sigma$ is an anti-automorphism of $K$ and $\varepsilon \in K$ is such that $\varepsilon^{\sigma} \varepsilon=1$ and $t^{\sigma^{2}}=\varepsilon t \varepsilon^{-1}$ for any $t \in K$. Following Buekenhout and Cohen [2, Chapter 10] we call $(\sigma, \varepsilon)$ an admissible pair of $K$. As in Tits [10, Chapter 8], we set

$$
K_{\sigma, \varepsilon}:=\left\{t-t^{\sigma} \varepsilon\right\}_{t \in K}, \quad K^{\sigma, \varepsilon}=\left\{t \in K \mid t=-t^{\sigma} \varepsilon\right\} .
$$

Clearly $K_{\sigma, \varepsilon}$ and $K^{\sigma, \varepsilon}$ are subgroups of the additive group of $K$. Moreover,

$$
\begin{gathered}
\lambda^{\sigma} K_{\sigma, \varepsilon} \lambda=K_{\sigma, \varepsilon} \text { and } \lambda^{\sigma} K^{\sigma, \varepsilon} \lambda=K^{\sigma, \varepsilon} \text { for every } \lambda \in K-\{0\}, \\
K_{\sigma, \varepsilon} \subseteq K^{\sigma, \varepsilon}, \\
K^{\sigma, \varepsilon}=K \text { if and only if } \sigma=\operatorname{id}_{K} \text { and } \varepsilon=-1, \\
K_{\sigma, \varepsilon}=K \text { if and only if } \sigma=\operatorname{id}_{K}, \varepsilon=-1 \text { and } \operatorname{char}(K) \neq 2 .
\end{gathered}
$$

The quotient group of the additive group of $K$ over $K_{\sigma, \varepsilon}$ is denoted by $K^{(\sigma, \varepsilon)}$ in [10]. In this paper we shall denote it by the symbol $\bar{K}$ :

$$
\bar{K}:=K^{(\sigma, \varepsilon)}=K / K_{\sigma, \varepsilon} .
$$

We will also adopt the following convention. Given $t \in K$ we denote by $\bar{t}$ the element of $\bar{K}$ represented by $t$ :

$$
\bar{t}:=t+K_{\sigma, \varepsilon} .
$$

Accordingly, $\overline{t+s}=t+s+K_{\sigma, \varepsilon}, \overline{t s}=t s+K_{\sigma, \varepsilon}$ and $\overline{0}$ is the null element of $\bar{K}$. If $X \subseteq K$ we put $\bar{X}:=\{\bar{t}\}_{t \in X}$. 


\subsubsection{Pairs of trace type}

Clearly, if $(\sigma, \varepsilon)$ is an admissible pair of a division ring $K$ then the pair $(\sigma,-\varepsilon)$ is also admissible. So, we can consider the groups $K_{\sigma,-\varepsilon}=\left\{t+t^{\sigma} \varepsilon\right\}_{t \in K}$ and $K^{\sigma,-\varepsilon}=\left\{t \in K \mid t=t^{\sigma} \varepsilon\right\}$. According to (2), $K_{\sigma,-\varepsilon} \subseteq K^{\sigma,-\varepsilon}$. Following Buekenhout and Cohen [2], when $K_{\sigma,-\varepsilon}=K^{\sigma,-\varepsilon}$ we say that the pair $(\sigma, \varepsilon)$ is of trace type. The following is well known (see Tits [10, Chapter 8], also Buekenhout and Cohen [2, Chapter 10]).

Lemma 2.1. Assume that either $\operatorname{char}(K) \neq 2$ or $\operatorname{char}(K)=2$ but $\sigma$ acts nontrivially on the center $Z(K)$ of $K$. Then, for every element $\varepsilon \in K$ forming an admissible pair with $\sigma$, the pair $(\sigma, \varepsilon)$ is of trace type.

\subsubsection{A scalar multiplication in the group $\bar{K}$}

According to (1), $\lambda^{\sigma} K_{\sigma, \varepsilon} \lambda=K_{\sigma, \varepsilon}$ for every $\lambda \in K$. So, we can define a scalar multiplication $\circ: \bar{K} \times K \rightarrow \bar{K}$ as follows: $\left(t+K_{\sigma, \varepsilon}\right) \circ \lambda=\lambda^{\sigma}\left(t+K_{\sigma, \varepsilon}\right) \lambda=$ $\lambda^{\sigma} t \lambda+K_{\sigma, \varepsilon}$, namely

$$
\bar{t} \circ \lambda=\overline{\lambda^{\sigma} t \lambda} \text { for any } \bar{t} \in \bar{K} \text { and } \lambda \in K \text {. }
$$

Clearly the following hold for any $\bar{t}, \bar{s} \in \bar{K}$ and $\lambda, \mu \in K$ :

$$
(\bar{t} \circ \lambda) \circ \mu=\bar{t} \circ(\lambda \mu) \text { and }(\overline{t+s}) \circ \lambda=\bar{t} \circ \lambda+\bar{s} \circ \lambda .
$$

Given an element $\bar{t} \in \bar{K}$ (a subset $\bar{H} \subseteq \bar{K}$ ) we put $\bar{t} \circ K:=\{\bar{t} \circ \lambda\}_{\lambda \in K}$ (respectively $\bar{H} \circ K:=\cup_{\bar{t} \in \bar{H}} \bar{t} \circ K$ ). We say that $\bar{t}$ is a $\circ$-vector if

$$
\bar{t} \circ(\lambda+\mu)=\bar{t} \circ \lambda+\bar{t} \circ \mu \text { for any } \lambda, \mu \in K .
$$

We denote by $\bar{K}^{\circ}$ the set of o-vectors of $\bar{K}$. It is easy to see that $\bar{K}^{\circ}+\bar{K}^{\circ} \subseteq \bar{K}^{\circ}$ and $\bar{K}^{\circ} \circ K \subseteq \bar{K}^{\circ}$. Moreover, $\overline{0} \in \bar{K}^{\circ}$ and $-\bar{K}^{\circ}=\bar{K}^{\circ}$. Thus, $\bar{K}^{\circ}$ can be regarded as a right $K$-vector space, with $\circ$ taken as the scalar multiplication.

All claims gathered in the next lemma are well known. Claim (1) is the same as Lemma 10.2.2 of Buekenhout and Cohen [2]. Claim (3) immediately follows from (1) while (2) follows from (1) and Lemma 2.1.

Lemma 2.2. All the following hold.

(1) $\bar{K}^{\circ}=K^{\sigma, \varepsilon} / K_{\sigma, \varepsilon}$.

(2) $\bar{K}^{\circ}=\{\overline{0}\}$ if and only if the pair $(\sigma, \varepsilon)$ is of trace type.

(3) $\bar{K}^{\circ}=\bar{K}$ if and only if $K^{\sigma, \varepsilon}=K$. 


\subsubsection{Closed subgroups of $\bar{K}$}

We say that a subgroup $\bar{H}$ of $\bar{K}$ is closed with respect to the scalar multiplication o defined above (also o-closed or just closed, for short) if $\bar{H} \circ K \subseteq \bar{H}$.

We refer the interested reader to Chapter 11 of Tits and Weiss [11] for a discussion of properties of closed subgroups. Here we only note that $\bar{K}$, the vector space $\bar{K}^{\circ}$ and all subspaces of $\bar{K}^{\circ}$ are closed subgroup of $\bar{K}$ and we mention the following, to be exploited in Section 3. Let $\bar{H}$ be a closed subgroup of $\bar{K}$. The scalar multiplication $\circ$ of $\bar{K}$ naturally induces a scalar multiplication on the quotient group $\bar{K} / \bar{H}$, which we shall denote by the same symbol $\circ$ used for the scalar multiplication of $\bar{K}$. Explicitly,

$$
(\bar{t}+\bar{H}) \circ \lambda:=\bar{t} \circ \lambda+\bar{H} \text { for every } \bar{t} \in \bar{K} .
$$

This definition is consistent, namely the coset $\bar{t} \circ \lambda+\bar{H}$ does not depend on the choice of the representative $\bar{t}$ of $\bar{t}+\bar{H}$. Moreover, if $\bar{H} \subseteq \bar{K}^{\circ}$ then $\bar{K}^{\circ} / \bar{H}$ is a $K$-vector space, with scalar multiplication $\circ$ defined as above.

\subsubsection{Proportionality of admissible pairs}

Given an admissible pair $(\sigma, \varepsilon)$ of $K$ and a nonzero scalar $\kappa \in K-\{0\}$, let $\varepsilon^{\prime}:=\kappa \kappa^{-\sigma} \varepsilon$ and let $\sigma^{\prime}$ be the anti-automorphism of $K$ defined as follows:

$$
t^{\sigma^{\prime}}:=\kappa t^{\sigma} \kappa^{-1} \text { for every } t \in K
$$

Both claims of the next lemma are well known (see Tits [10, Chapter 8]):

Lemma 2.3. The pair $\left(\sigma^{\prime}, \varepsilon^{\prime}\right)$ is admissible. Moreover:

(1) $\kappa K_{\sigma, \varepsilon}=K_{\sigma^{\prime}, \varepsilon^{\prime}}$ and $\kappa K^{\sigma, \varepsilon}=K^{\sigma^{\prime}, \varepsilon^{\prime}}$.

(2) $\kappa \lambda^{\sigma} t \lambda=\lambda^{\sigma^{\prime}} \kappa t \lambda$ for any $t \in K$.

By (1) of Lemma 2.3, left multiplication by $\kappa$ induces a group isomorphism from $K / K_{\sigma, \varepsilon}$ to $K / K_{\sigma^{\prime}, \varepsilon^{\prime}}$ as well as from $K^{\sigma, \varepsilon} / K_{\sigma, \varepsilon}$ to $K^{\sigma^{\prime}, \varepsilon^{\prime}} / K_{\sigma^{\prime}, \varepsilon^{\prime}}$.

When dealing with two pairs $(\sigma, \varepsilon)$ and $\left(\sigma^{\prime}, \varepsilon^{\prime}\right)$ as above it is convenient to keep a record of them in our notation. So we put $\bar{K}^{\sigma, \varepsilon}=K / K_{\sigma, \varepsilon}, \bar{K}^{\sigma^{\prime}, \varepsilon^{\prime}}=$ $K / K_{\sigma^{\prime}, \varepsilon^{\prime}}, \bar{K}^{\circ, \sigma, \varepsilon}=K^{\sigma, \varepsilon} / K_{\sigma, \varepsilon}, \bar{K}^{\circ, \sigma^{\prime}, \varepsilon^{\prime}}=K^{\sigma^{\prime}, \varepsilon^{\prime}} / K_{\sigma^{\prime}, \varepsilon^{\prime}}, \bar{t}^{\sigma, \varepsilon}=t+K_{\sigma, \varepsilon}, \bar{t}^{\sigma^{\prime}, \varepsilon^{\prime}}=$ $t+K_{\sigma^{\prime}, \varepsilon^{\prime}}$ and we denote the scalar multiplications of $\bar{K}^{\sigma, \varepsilon}$ and $\bar{K}^{\sigma^{\prime}, \varepsilon^{\prime}}$ by the symbols $\circ_{\sigma}$ and $\circ_{\sigma^{\prime}}$ respectively. This notation is admittedly rather clumsy. We will avoid it as far as possible, but in the present context we need it. With the above notation, claim (2) of Lemma 2.3 can be rewritten as follows:

$$
\kappa\left(\bar{t}^{\sigma, \varepsilon} \circ_{\sigma} \lambda\right)=\left(\kappa\left(\bar{t}^{\sigma, \varepsilon}\right)\right) \circ_{\sigma^{\prime}} \lambda=\left(\overline{(\kappa t)}^{\sigma^{\prime}, \varepsilon^{\prime}}\right) \circ_{\sigma^{\prime}} \lambda .
$$


Thus, left multiplication by $\kappa$ is an isomorphism of $K$-vector spaces from $\bar{K}^{\circ, \sigma, \varepsilon}$ to $\bar{K}^{\circ, \sigma^{\prime}, \varepsilon^{\prime}}$. With $\kappa,(\sigma, \varepsilon)$ and $\left(\sigma^{\prime}, \varepsilon^{\prime}\right)$ as in $(11)$, we write $\left(\sigma^{\prime}, \varepsilon^{\prime}\right)=\kappa \cdot(\sigma, \varepsilon)$ and we say that the pairs $(\sigma, \varepsilon)$ and $\left(\sigma^{\prime}, \varepsilon^{\prime}\right)$ are proportional.

Clearly, if $\left(\sigma^{\prime}, \varepsilon^{\prime}\right)=\kappa \cdot(\sigma, \varepsilon)$ then $(\sigma, \varepsilon)=\kappa^{-1} \cdot\left(\sigma^{\prime}, \varepsilon^{\prime}\right)$. If moreover $\left(\sigma^{\prime \prime}, \varepsilon^{\prime \prime}\right)=$ $\kappa^{\prime} \cdot(\sigma, \varepsilon)$ then $\left(\sigma^{\prime \prime}, \varepsilon^{\prime \prime}\right)=\left(\kappa^{\prime} \kappa\right) \cdot(\sigma, \varepsilon)$. It is also clear that $\kappa \cdot(\sigma, \varepsilon)=(\sigma, \varepsilon)$ if and only if $\kappa \in Z(K)$ and $\kappa^{\sigma}=\kappa$.

\subsection{Reflexive sesquilinear forms}

Given a division ring $K$, a left $K$-vector space $V$ and an anti-automorphism $\sigma$ of $K$, a $\sigma$-sesquilinear form is a mapping $f: V \times V \rightarrow K$ such that

$$
\begin{aligned}
& f\left(x_{1} \lambda_{1}+x_{2} \lambda_{2}, y_{1} \mu_{1}+y_{2} \mu_{2}\right) \\
& \quad=\lambda_{1}^{\sigma} f\left(x_{1}, y_{1}\right) \mu_{1}+\lambda_{1}^{\sigma} f\left(x_{1}, y_{2}\right) \mu_{2}+\lambda_{2}^{\sigma} f\left(x_{2}, y_{1}\right) \mu_{1}+\lambda_{2}^{\sigma} f\left(x_{2}, y_{2}\right) \mu_{2}
\end{aligned}
$$

for all $x_{1}, x_{2}, y_{1}, y_{2} \in V$ and $\lambda_{1}, \lambda_{2}, \mu_{1}, \mu_{2} \in K$. We say that $f$ is trivial when $f(x, y)=0$ for any choice of $x, y \in V$. Obviously, if $f$ is non-trivial then $\sigma$ is uniquely determined by (12). When $\sigma=\mathrm{id}_{K}$ (whence $K$ is a field, namely it is commutative) then $f$ is said to be bilinear.

A sesquilinear form $f$ is said to be reflexive if, for any choice of $x, y \in V$, we have $f(x, y)=0$ if and only if $f(y, x)=0$. It is well known (Tits [10, Chapter 8]) that a non-trivial $\sigma$-sesquilinear form is reflexive if and only if there exists a (uniquely determined) element $\varepsilon \in K$ such that

$$
f(y, x)=f(x, y)^{\sigma} \varepsilon \text { for all } x, y \in V .
$$

If this is the case then $(\sigma, \varepsilon)$ is an admissible pair and $f$ is called a $(\sigma, \varepsilon)$-sesquilinear form. A symmetric bilinear form is an $\left(\mathrm{id}_{K}, 1\right)$-sesquilinear form. A bilinear form $f$ is said to be alternating if

$$
f(x, x)=0 \text { for any } x \in V .
$$

Non-trivial alternating forms are $\left(\mathrm{id}_{K},-1\right)$-sesquilinear. Conversely, if $K$ is a field of characteristic $\operatorname{char}(K) \neq 2$ then all $\left(\operatorname{id}_{K},-1\right)$-sesquilinear forms are alternating. On the other hand, let $\operatorname{char}(K)=2$. Then $1=-1$. In this case an $\left(\operatorname{id}_{K},-1\right)$-sesquilinear form is just a symmetric bilinear form. Obviously, not all symmetric bilinear forms satisfy (14).

Let $f: V \times V \rightarrow K$ be a $(\sigma, \varepsilon)$-sesquilinear form. By (13), $f(x, x) \in K^{\sigma,-\varepsilon}$ for every $x \in V$. The form $f$ is said to be trace-valued if $f(x, x) \in K_{\sigma,-\varepsilon}$ for every $x \in V$. Clearly, if the pair $(\sigma, \varepsilon)$ is of trace type then all $(\sigma, \varepsilon)$-sesquilinear forms are trace-valued. Hence, by Lemma 2.1, when either $\operatorname{char}(K) \neq 2$ or 
$\operatorname{char}(K)=2$ but $\sigma$ acts non-trivially on $Z(K)$, all $(\sigma, \varepsilon)$-sesquilinear forms are trace-valued. When $K$ is a field of characteristic 2 the pair $\left(\mathrm{id}_{K}, 1\right)$ is not of trace type. In this case an $\left(\mathrm{id}_{K}, 1\right)$-sesquilinear form is trace-valued if and only if it is alternating.

The following characterization of trace-valued sesquilinear forms is well known (Tits [10, Chapter 8], also Buekenhout and Cohen [2, Chapter 10]).

Lemma 2.4. $A(\sigma, \varepsilon)$-sesquilinear form $f: V \times V \rightarrow K$ is trace-valued if and only if there exists a $\sigma$-sesquilinear form $g: V \times V \rightarrow K$ such that $f(x, y)=$ $g(x, y)+g(y, x)^{\sigma} \varepsilon$ for all $x, y \in V$.

\subsubsection{Orthogonality and the polar space $S_{f}$}

Given a $(\sigma, \varepsilon)$-sesquilinear form $f: V \times V \rightarrow K$, we say that two vectors $x, y \in K$ are orthogonal (with respect to $f$ ) if $f(x, y)=0$. If $x$ and $y$ are orthogonal then we write $x \perp y$. Given a vector $x \in V$ we put $x^{\perp}:=\{y \in V \mid y \perp x\}$ and, for a subset $X \subseteq V$, we set $X^{\perp}:=\bigcap_{x \in X} x^{\perp}$. Clearly $x^{\perp}$ is either a hyperplane or the whole of $V$. Hence $X^{\perp}$ is a subspace of $V$, for any $X \subseteq V$. We set

$$
\operatorname{Rad}(f):=V^{\perp}=\left\{x \in V \mid x^{\perp}=V\right\}
$$

and we call $\operatorname{Rad}(f)$ the radical of $f$. We say that $f$ is degenerate if $\operatorname{Rad}(f) \neq\{0\}$.

A vector $x \in V$ is said to be isotropic for $f$ (also $f$-isotropic) if $f(x, x)=0$, namely $x \in x^{\perp}$. A subset $X \subseteq V$ is totally isotropic for $f$ (totally $f$-isotropic) if $X \subseteq X^{\perp}$. Clearly, $\operatorname{Rad}(f)$ is a totally isotropic subspace of $V$. We say that the form $f$ is strictly isotropic if it admits at least one isotropic vector $x \notin \operatorname{Rad}(f)$. The following is well known (Tits [10, Chapter 8], Buekenhout and Cohen [2, Chapter 10]).

Proposition 2.5. Let $f: V \times V \rightarrow K$ be a strictly isotropic $(\sigma, \varepsilon)$-sesquilinear form. Then $f$ is trace-valued if and only if $V$ is spanned by the set of $f$-isotropic vectors.

As in Subsection 1.3.2, given a non-zero vector $x \in V$ we denote by $[x]$ the point of $\mathrm{PG}(V)$ represented by the vector $x$ and, for a subspace $X$ of $V$, we set $[X]=\{[x]\}_{x \in X-\{0\}}$. We also write $\left[x_{1}, x_{2}, \ldots, x_{k}\right]$ for $\left[\left\langle x_{1}, x_{2}, \ldots, x_{k}\right\rangle\right]$.

Given a $(\sigma, \varepsilon)$-sesquilinear form $f: V \times V \rightarrow K$, a point $[x]$ of $\mathrm{PG}(V)$ is said to be isotropic for $f$ (also $f$-isotropic) if the vector $x$ is $f$-isotropic. Similarly, given a subspace $X$ of $V$, the subspace $[X]$ of $\mathrm{PG}(V)$ is totally isotropic for $f$ (totally $f$-isotropic) if $X$ is totally $f$-isotropic. We denote by $P_{f}$ and $L_{f}$ the set of $f$-isotropic points and totally $f$-isotropic lines of $\mathrm{PG}(V)$ and we put $S_{f}:=$ $\left(P_{f}, L_{f}\right)$. 
Assume that $P_{f} \neq \emptyset \neq L_{f}$. Then $S_{f}$ is a polar space (Buekenhout and Cohen [2, Chapter 7]). We call it the polar space associated to $f$. The singular subspaces of $S_{f}$ are the totally $f$-isotropic subspaces of $\mathrm{PG}(V)$. The subspace $[\operatorname{Rad}(f)]$ is the radical of $S_{f}$. So, $S_{f}$ is non-degenerate if and only if $f$ is nondegenerate. The set $P_{f}$ spans $\operatorname{PG}(V)$ if and only if $f$ is either trivial or tracevalued (Proposition 2.5).

Let $e_{f}: S_{f} \rightarrow \mathrm{PG}(V)$ be the inclusion mapping of $S_{f}$ in $\mathrm{PG}(V)$. If $P_{f}$ spans $\mathrm{PG}(V)$ then $e_{f}$ is a projective embedding in the sense of Subsection 1.3.3.

\subsubsection{Proportionality of reflexive sesquilinear forms}

Let $f: V \times V \rightarrow K$ be a non-trivial $(\sigma, \varepsilon)$-sesquilinear form and let $\kappa \in K-\{0\}$. It is well known (Tits [10, Chapter 8]) that $\kappa f$ is a $\left(\sigma^{\prime}, \varepsilon^{\prime}\right)$-sesquilinear form where $\left(\sigma^{\prime}, \varepsilon^{\prime}\right)=\kappa \cdot(\sigma, \varepsilon)$ (notation as in Subsection 2.1.4). We say that $f$ and $f^{\prime}$ are proportional.

Clearly, proportional reflexive sesquilinear forms define the same orthogonality relation. A partial converse of this fact also holds, but in order to state it we need one more definition: the non-degenerate rank of a polar space $S$ is the rank of the quotient of $S$ over its radical (Buekenhout and Cohen [2, 7.5.1]). The next proposition is implicit in the theory developed in Chapter 9 of Buekenhout and Cohen [2].

Proposition 2.6. For $i=1,2$, let $\left(\sigma_{i}, \varepsilon_{i}\right)$ be an admissible pair of $K$ and let $f_{i}: V \times V \rightarrow K$ be a $\left(\sigma_{i}, \varepsilon_{i}\right)$-sesquilinear form. Suppose that $\mathrm{PG}(V)$ admits a full subgeometry $S=(P, L)$ such that $S$ is a polar space with non-degenerate rank at least 2, it is a subspace of either $S_{f_{1}}$ and $S_{f_{2}}$ and the point-set $P$ of $S$ spans $\mathrm{PG}(V)$. Then the forms $f_{1}$ and $f_{2}$ are proportional.

In particular, if $S_{f_{1}}=S_{f_{2}}$ and the polar space $S:=S_{f_{1}}=S_{f_{2}}$ has nondegenerate rank at least 2 , then $f_{1}$ and $f_{2}$ are proportional.

\subsection{Pseudo-quadratic forms}

Given a division ring $K$ and an admissible pair $(\sigma, \varepsilon)$ of $K$, let $\bar{K}=K^{(\sigma, \varepsilon)}$, as in (5) of Subsection 2.1. The scalar multiplication $\circ$ is defined as in (7) and, for $t \in K$, we write $\bar{t}$ for $t+K_{\sigma, \varepsilon}$, as in Subsection 2.1.

Let $V$ be a right $K$-vector space. A $(\sigma, \varepsilon)$-quadratic form on $V$ (also called a pseudo-quadratic form) is a map $q: V \rightarrow \bar{K}$ such that

$q(x \lambda)=q(x) \circ \lambda$ for any $x \in V$ and $\lambda \in K$; 
(Q2) a trace-valued $(\sigma, \varepsilon)$-sesquilinear form $f: V \times V \rightarrow K$ exists such that

$$
q(x+y)=q(x)+q(y)+\overline{f(x, y)} \text { for any choice of } x, y \in V .
$$

We call $f$ a sesquilinearization of $q$. Note that in the above definition we allow $\bar{K}=\{\overline{0}\}$ (namely $K_{\sigma, \varepsilon}=K$ ), but we warn that when $\bar{K}=\{\overline{0}\}$ both conditions (Q1) and (Q2) are vacuous. In particular, when $\bar{K}=\{\overline{0}\}$ every trace-valued $(\sigma, \varepsilon)$-sesquilinear form satisfies (Q2). On the other hand (Tits [10, Chapter 8]):

Lemma 2.7. Let $\bar{K} \neq\{\overline{0}\}$. Then $q$ admits a unique sesquilinearization.

We say that a pseudo-quadratic form $q$ is trivial if $q(x)=\overline{0}$ for any $x \in V$. Clearly, if $\bar{K}=\{\overline{0}\}$ then $q$ is trivial. Finally, we warn that $\left(\mathrm{id}_{K}, 1\right)$-quadratic forms are usually called quadratic forms, for short. In this paper we shall conform to that habit.

Remark 2.8. In the literature, pseudo-quadratic forms are defined only when $\bar{K} \neq\{\overline{0}\}$. However, in the theory of generalized pseudo-quadratic forms, to be exposed in Section 3, we shall allow forms with trivial codomain. Accordingly, we have allowed $\bar{K}=\{\overline{0}\}$ here.

\subsubsection{Facilitating forms}

Every $(\sigma, \varepsilon)$-quadratic form $q$ admits a so-called facilitating form, namely a $\sigma$ sesquilinear form $g: V \times V \rightarrow K$ such that

$$
q(x)=\overline{g(x, x)} \text { for any } x \in V .
$$

If $\bar{K}=\overline{0}$ every $\sigma$-sesquilinear form is a facilitating form for $q$. Let $\bar{K} \neq \overline{0}$ and let $f$ be the sesquilinearization of $q$. Then all facilitating forms of $q$ are obtained as follows (Tits [10, Chapter 8]). Let $\left(e_{i}\right)_{i \in I}$ be a basis of $V$. Assume that a total ordering $<$ is given on the index set $I$. For every $i \in I$ let $g_{i} \in K$ be such that $q\left(e_{i}\right)=\bar{g}_{i}$. For any two vectors $x=\sum_{i \in I} e_{i} \lambda_{i}$ and $y=\sum_{i \in I} e_{i} \mu_{i}$ of $V$, put

$$
g(x, y):=\sum_{i<j} \lambda_{i}^{\sigma} f\left(e_{i}, e_{j}\right) \mu_{j}+\sum_{i \in I} \lambda_{i}^{\sigma} g_{i} \mu_{i} .
$$

(Note that all sums occurring in (16) are well defined, since only finitely many of the scalars $\lambda_{i}$ and $\mu_{i}$ are different from 0 .) Then the mapping $g$ defined as in (16) is a facilitating form for $q$. Moreover,

$$
f(x, y)=g(x, y)+g(y, x)^{\sigma} \varepsilon \text { for any } x, y \in V .
$$


Conversely, given a $\sigma$-sesquilinear form $g: V \times V \rightarrow K$ and an element $\varepsilon \in K$ forming an admissible pair with $\sigma$, let $q: V \rightarrow \bar{K}$ be defined as in (15). Then $q$ is a $(\sigma, \varepsilon)$-quadratic form and the form $f$ defined as in (17) is the sesquilinearization of $q$. Note that $f$ is indeed trace-valued, by Lemma 2.4.

\subsubsection{The polar space $S_{q}$}

Let $q: V \rightarrow \bar{K}$ be a $(\sigma, \varepsilon)$-quadratic form. A vector $x \in V$ is said to be singular for $q$ (also $q$-singular) if $q(x)=\overline{0}$. A subspace $X \subset V$ is totally singular for $q$ (also totally $q$-singular) if $q(x)=\overline{0}$ for every $x \in X$.

Clearly, if $q(x)=\overline{0}$ for a vector $x \in V$ then $q(x \lambda)=\overline{0}$ for any $\lambda \in K$. Therefore a point $[x]$ of $\mathrm{PG}(V)$ is totally $q$-singular as a 1-dimensional subspace of $V$ if and only if $x$ is $q$-singular. If this is the case then we say that the point $[x]$ is singular for $q$ (also $q$-singular). A subspace $[X]$ of $\mathrm{PG}(V)$ is said to be totally singular for $q$ (also totally $q$-singular) if all of its points are $q$-singular.

We denote by $P_{q}$ and $L_{q}$ the set of $q$-singular points and totally $q$-singular lines of $\mathrm{PG}(V)$ and we put $S_{q}:=\left(P_{q}, L_{q}\right)$. Note that $P_{q}$ or $L_{q}$ could be empty. The opposite situation, where $S_{q}=\operatorname{PG}(V)$, occurs when $q$ is trivial.

For the rest of this subsection we assume that $P_{q} \neq \emptyset \neq L_{q}$ and $\bar{K} \neq \overline{0}$. We denote by $f$ the sesquilinearization of $q$. All propositions to be stated in the rest of this subsection are well known. Their proofs can be found in Tits [10, Chapter 8] and Buekenhout and Cohen [2, Chapter 10]. However we shall recall those proofs here, since in Section 3 we will need them for reference.

Proposition 2.9. The point-line geometry $S_{q}=\left(P_{q}, L_{q}\right)$ is a subspace of the polar space $S_{f}$ associated to $f$. Explicitly:

(1) $P_{q} \subseteq P_{f}$;

(2) a projective line $[x, y]$ belongs to $L_{q}$ if and only if $q(x)=q(y)=\overline{0}$ and $f(x, y)=0$.

Proof. This is one of the proofs we want to recall in view of Section 3. Let $q(x)=\overline{0}$. Then $q(x(\lambda+\mu))=\overline{0}$ as well, for any choice of scalars $\lambda, \mu \in K$. It follows from (Q2) with $x$ and $y$ replaced by $x \lambda$ and $x \mu$ respectively that $\lambda^{\sigma} f(x, x) \mu \in K_{\sigma, \varepsilon}$. If $f(x, x) \neq 0$ the arbitrariness of $\lambda$ and $\mu$ forces $K_{\sigma, \varepsilon}=K$, contradicting the assumption that $\bar{K} \neq \overline{0}$. Therefore $f(x, x)=0$. Claim (1) is proved.

Turning to claim (2), let $[x, y] \in L_{q}$. Then $q(x \lambda+y \mu)=\overline{0}$ for any choice of $\lambda, \mu \in K$. According to (Q2), this forces $\lambda^{\sigma} f(x, y) \mu \in K_{\sigma, \varepsilon}$. Hence $f(x, y)=0$, since $K_{\sigma, \varepsilon} \subset K$. The 'only if' part of (2) is proved. The 'if' part is trivial. 
By Proposition 2.9, a subspace $\left[x_{1}, x_{2}, \ldots, x_{k}\right]$ of $\mathrm{PG}(V)$ is totally $q$-singular if and only if it is totally isotropic for $f$ and $q\left(x_{1}\right)=q\left(x_{2}\right)=\cdots=q\left(x_{k}\right)=\overline{0}$. Moreover:

Corollary 2.10. The point-line geometry $S_{q}$ is a polar space. Its singular subspaces are the totally $q$-singular subspaces of $\operatorname{PG}(V)$. The set $P_{q} \cap[\operatorname{Rad}(f)]$ is the radical of $S_{q}$.

The radical $P_{q} \cap[\operatorname{Rad}(f)]$ of $S_{q}$ is a subspace of $[\operatorname{Rad}(f)]$. We call it the radical of $q$ and we denote it by the symbol $\operatorname{Rad}(q)$. Following Buekenhout and Cohen [2, Chapter 10] and Tits and Weiss [11, Chapter 11], we call $\operatorname{Rad}(f)$ the defect of $q$ (but we warn that this word is used with a different meaning in Tits [10]). The form $q$ is said to be singular (also degenerate) if $\operatorname{Rad}(q) \neq\{0\}$.

If $P_{q}$ spans $\mathrm{PG}(V)$ then the inclusion mapping $e_{q}: S_{q} \rightarrow \mathrm{PG}(V)$ is a projective embedding in the sense of Subsection 1.3.3. A sufficient condition for $P_{q}$ to span $\mathrm{PG}(V)$ is given by the next proposition.

Proposition 2.11. If $P_{q} \nsubseteq[\operatorname{Rad}(f)]$ then $P_{q}$ spans $\mathrm{PG}(V)$.

Proof. In view of Section 3, we also give a sketch of this proof. Suppose that $P_{q} \not \subset[\operatorname{Rad}(f)]$. Then there exists a $q$-singular point $[a] \notin[\operatorname{Rad}(f)]$. As $a \notin$ $\operatorname{Rad}(f)$, the space $a^{\perp}$ is a hyperplane of $V$. Let $l=[a, b]$ be a projective line of $\operatorname{PG}(V)$ through $[a]$ not contained in $\left[a^{\perp}\right]$. Then $f(a, b) \neq 0$. Moreover,

$$
q(a \lambda+b)=q(a) \circ \lambda+q(b)+\overline{\lambda^{\sigma} f(a, b)}=q(b)+\overline{\lambda^{\sigma} f(a, b)}
$$

by (Q2) and since $q(a)=\overline{0}$. As $f(a, b) \neq 0$, there exists a scalar $\lambda \in K$ such that $q(b)+\overline{\lambda^{\sigma} f(a, b)}=\overline{0}$. Then $q(a \lambda+b)=\overline{0}$ by (18). So, the vector $b_{l}:=a \lambda+b$ is $q$-singular and $\left[b_{l}\right] \neq[a]$.

Let $\Lambda_{a}$ be the set of lines of $\mathrm{PG}(V)$ that contain $[a]$ but are not contained in $\left[a^{\perp}\right]$. By the previous paragraph, every line $l \in \Lambda_{a}$ contains a $q$-singular point $\left[b_{l}\right] \neq[a]$. Let $\Pi_{a}:=\left\{\left[b_{l}\right]\right\}_{l \in \Lambda_{a}}$. Then $\Pi_{a}$ is contained in $P_{q}$ and spans $\operatorname{PG}(V)$. Hence $\left\langle P_{q}\right\rangle=\mathrm{PG}(V)$.

We know that $S_{q}$ is a subspace of $S_{f}$ (Proposition 2.9), but it could be a proper subspace of $S_{f}$, namely the equality $f(x, x)=0$ does not force $q(x)=\overline{0}$. The following is all we can say in general about $q(x)$ when $f(x, x)=0$.

Lemma 2.12. For $x \in V$, if $f(x, x)=0$ then $q(x) \in \bar{K}^{\circ}$.

Proof. We will give a sketch of this proof, too. Recall that $\bar{K}^{\circ}=K^{\sigma, \varepsilon} / K_{\sigma, \varepsilon}$ (Lemma 2.2(1)). Let $f(x, x)=0$. Then

$$
\begin{aligned}
q(x) \circ(\lambda+\mu) & =q(x(\lambda+\mu))=q(x \lambda)+q(x \mu)+\overline{\lambda^{\sigma} f(x, x) \mu} \\
& =q(x \lambda)+q(x \mu)=q(x) \circ \lambda+q(x) \circ \mu .
\end{aligned}
$$


Let $t \in K$ be such that $q(x)=\bar{t}$. By the above,

$$
(\lambda+\mu)^{\sigma} t(\lambda+\mu) \equiv \lambda^{\sigma} t \lambda+\mu^{\sigma} t \mu\left(\bmod K_{\sigma, \varepsilon}\right) .
$$

Hence $\lambda^{\sigma} t \mu+\mu^{\sigma} t \lambda \in K_{\sigma, \varepsilon}$. Recalling that $\lambda^{\sigma} t \mu-\left(\lambda^{\sigma} t \mu\right)^{\sigma} \varepsilon \in K_{\sigma, \varepsilon}$ and $\left(\lambda^{\sigma} t \mu\right)^{\sigma} \varepsilon=$ $\mu^{\sigma} t^{\sigma} \varepsilon \lambda$, we obtain that $\mu^{\sigma} t \lambda+\mu^{\sigma} t^{\sigma} \varepsilon \lambda \in K_{\sigma, \varepsilon}$, namely

$$
\mu^{\sigma}\left(t+t^{\sigma} \varepsilon\right) \lambda \in K_{\sigma, \varepsilon} .
$$

Since $K_{\sigma, \varepsilon} \neq K$ by assumption and (19) holds for any choice of $\lambda, \mu \in K$, it follows that $t+t^{\sigma} \varepsilon=0$, namely $t \in K^{\sigma, \epsilon}$. Hence $\bar{t} \in K^{\sigma, \varepsilon} / K_{\sigma, \varepsilon}=\bar{K}^{\circ}$.

Proposition 2.13. Let $(\sigma, \varepsilon)$ be of trace type. Then $S_{q}=S_{f}$.

Proof. Let $(\sigma, \varepsilon)$ be of trace type. Then $\bar{K}^{\circ}=\overline{0}$ by claim (2) of Lemma 2.2. The conclusion follows from Lemma 2.12.

\subsubsection{Proportionality of pseudo-quadratic forms}

In this subsection we adopt the notation of Subsection 2.1.4, thus denoting the group $\bar{K}=K / K_{\sigma, \varepsilon}$ by the symbol $\bar{K}^{\sigma, \varepsilon}$. Assuming $K_{\sigma, \varepsilon} \neq K$, let $q: V \rightarrow \bar{K}^{\sigma, \varepsilon}$ be a non-trivial $(\sigma, \varepsilon)$-quadratic form and let $f$ be its sesquilinearization. Given a scalar $\kappa \in K-\{0\}$, let $\left(\sigma_{\kappa}, \varepsilon_{\kappa}\right):=\kappa \cdot(\sigma, \varepsilon)$. Let $\kappa q: V \rightarrow \bar{K}^{\sigma_{\kappa}, \varepsilon_{\kappa}}$ be the function mapping every $x \in V$ onto $\kappa q(x) \in \bar{K}^{\sigma_{\kappa}, \varepsilon_{\kappa}}$ (well defined by Lemma 2.3). Then $\kappa q$ is a $\left(\sigma_{\kappa}, \varepsilon_{\kappa}\right)$-quadratic form and $\kappa f$ is the sesquilinearization of $\kappa q$ (Tits [10, Chapter 8]). Clearly, $S_{\kappa q}=S_{q}$. We say that $q$ and $\kappa q$ are proportional.

Proposition 2.14. For $i=1,2$, let $q_{i}: V \rightarrow \bar{K}^{\sigma_{i}, \varepsilon_{i}}$ be a non-trivial $\left(\sigma_{i}, \varepsilon_{i}\right)$ quadratic form such that $S_{q_{i}}$ has non-degenerate rank at least 2. Suppose that $S_{q_{1}}=S_{q_{2}}$. Then $q_{1}$ and $q_{2}$ are proportional.

Proof. This proposition is well known (see e.g. Tits [10, Chapter 8]). Nevertheless we give a sketch of the proof here, since in Section 3 we will need it for reference.

Let $f_{1}$ and $f_{2}$ be the sesquilinearizations of $q_{1}$ and $q_{2}$. By Proposition 2.11, the set $P_{q_{i}}$ spans $\mathrm{PG}(V)$. for $i=1,2$. Moreover $S_{q_{i}}$ is a subspace of $S_{f_{i}}$. By assumption, the polar space $S_{q_{i}}$ has non-degenerate rank at least 2 . Hence the equality $S_{q_{1}}=S_{q_{2}}$ forces $f_{1}$ and $f_{2}$ to be proportional, by Proposition 2.6. It follows that $q_{1}$ and $q_{2}$ admit proportional facilitating forms (defined by equation (16), applied to a basis of singular vectors). Hence they are proportional. 


\section{Generalized pseudo-quadratic forms}

\subsection{Definition}

Given a division ring $K$ and an admissible pair $(\sigma, \varepsilon)$ of $K$, let $\bar{R}$ be a o-closed subgroup of $\bar{K}$ (see Subsection 2.1.2). We denote by $R$ the pre-image of $\bar{R}$ under the projection $t \mapsto \bar{t}=t+K_{\sigma, \varepsilon}$ of $K$ onto $\bar{K}=K / K_{\sigma, \varepsilon}$, namely:

$$
R:=\{t \mid \bar{t} \in \bar{R}\} .
$$

We recall that a scalar multiplication is induced by $\circ$ on the factor group $\bar{K} / \bar{R}$, as explained in (10). Clearly $\bar{R}$ is the null element of $\bar{K} / \bar{R}$. When $\bar{R}$ is given this role, we denote it by the symbol $0_{\bar{R}}$.

Given a $K$-vector space $V$, a generalized $(\sigma, \varepsilon)$-quadratic form (also generalized pseudo-quadratic form) is a map $q: V \rightarrow \bar{K} / \bar{R}$ such that

(Q'1) $\quad q(x \lambda)=q(x) \circ \lambda$ for any $x \in V$ and $\lambda \in K$;

(Q'2) a trace-valued $(\sigma, \varepsilon)$-sesquilinear form $f: V \times V \rightarrow K$ exists such that

$$
q(x+y)=q(x)+q(y)+(\overline{f(x, y)}+\bar{R}) ? ? ? \text { for all } x, y \in V .
$$

We call $\bar{R}$ the co-defect of $q$. Thus, a pseudo-quadratic form is just a generalized pseudo-quadratic form with trivial co-defect.

Remark 3.1. In Subsection 4.2.2 we will show that the co-defect $\bar{R}$ of $q$ is involved as a summand in the defect of a suitable pseudo-quadratic form, called the dominant cover of $q$. This is a motivation for calling $\bar{R}$ the co-defect of $q$.

A sesquilinear form $f$ as in (Q'2) is called a sesquilinearization of $q$. The next lemma is a generalization of Lemma 2.7. Claim (2) of this lemma is obvious. Claim (1) can be proved by the same argument used for pseudo-quadratic forms in [10], but for replacing $K_{\sigma, \varepsilon}$ with the group $R$ defined in (20). (See also Tits and Weiss $[11,11.19]$.)

Lemma 3.2. Let $q: V \rightarrow \bar{K} / \bar{R}$ be a generalized pseudo-quadratic form.

(1) If $\bar{R} \neq \bar{K}$ then q admits exactly one sesquilinearization.

(2) Let $\bar{R}=\bar{K}$. Then every trace-valued $(\sigma, \varepsilon)$-sesquilinear form on $V$ is a sesquilinearization of $q$.

Every generalized $(\sigma, \varepsilon)$-quadratic form also admits a facilitating form, namely a $\sigma$-sesquilinear form $g: V \times V \rightarrow K$ such that

$$
q(x)=\overline{g(x, x)}+\bar{R} \text { for any } x \in V .
$$


If $\bar{R}=\bar{K}$ then every $\sigma$-sesquilinear form is a facilitating form for $q$. Let $\bar{R} \neq \bar{K}$ and let $f$ be the sesquilinearization of $q$. It is straightforward to prove that all facilitating forms of $q$ are obtained as follows (compare [11, 11.29]). Let $\left(e_{i}\right)_{i \in I}$ be a basis of $V$ and $<$ a total ordering of $I$. For every $i \in I$ let $g_{i} \in K$ be such that $q\left(e_{i}\right)=\bar{g}_{i}+\bar{R}$. For $x, y \in V$ let $g(x, y)$ be defined as in (16). Then $g$ is a facilitating form for $q$. Moreover $f(x, y)=g(x, y)+g(y, x)^{\sigma} \varepsilon$, as in (17).

Conversely, given a $\sigma$-sesquilinear form $g: V \times V \rightarrow K$ and an element $\varepsilon \in K$ forming an admissible pair with $\sigma$, let $q: V \rightarrow \bar{K}$ be defined as in (21). Then $q$ is a generalized $(\sigma, \varepsilon)$-quadratic form and the form $f$ defined as in (17) is the sesquilinearization of $q$.

\subsection{Basic properties}

In this subsection and the following ones we shall discuss properties of generalized pseudoquadratic forms. Many (but not all) of them are straightforward generalizations of analogous properties of pseudo-quadratic forms. We begin with the following theorem.

Theorem 3.3. Let $\bar{R} \neq \bar{K}$. Let $q: V \rightarrow \bar{K} / \bar{R}$ be a generalized $(\sigma, \varepsilon)$-quadratic form, let $f$ be its sesquilinearization and let $R$ be as in (20). Then all the following hold:

(1) $\bar{R} \subseteq \bar{K}^{\circ}$. In other words, $\bar{R}$ is a vector subspace of $\bar{K}^{\circ}$.

(2) For every vector $x \in V$, if $q(x)=0_{\bar{R}}$ then $f(x, x)=0$.

(3) Let $x \in V$ be such that $f(x, x)=0$. Then $q(x) \in \bar{K}^{\circ} / \bar{R}$ (well defined in view of claim (1)).

Proof. In view of (Q'1) and (Q'2), we have

$$
q(x) \circ(\lambda+\mu)+\bar{R}=q(x(\lambda+\mu))=q(x) \circ \lambda+q(x) \circ \mu+\lambda^{\sigma} f(x, x) \mu
$$

for any choice of $\lambda, \mu \in K$. Therefore, given $t \in K$ such that $\bar{t}+\bar{R}=q(x)$, we have $\lambda^{\sigma} t \mu+\mu^{\sigma} t \lambda-\lambda^{\sigma} f(x, x) \mu \in R$. As $K_{\sigma, \varepsilon} \subseteq R$ and $\mu^{\sigma} t \lambda-\lambda^{\sigma} t^{\sigma} \varepsilon \mu=$ $\mu^{\sigma} t \lambda-\left(\mu^{\sigma} t \lambda\right)^{\sigma} \varepsilon \in K_{\sigma, \varepsilon}$ we obtain that $\lambda^{\sigma} t \mu+\lambda^{\sigma} t^{\sigma} \varepsilon \mu-\lambda^{\sigma} f(x, x) \mu \in R$, namely

$$
\lambda^{\sigma}\left(t+t^{\sigma} \varepsilon-f(x, x)\right) \mu \in R \text { for any choice of } \lambda, \mu \in K .
$$

As $R \neq K$ by assumption, (22) forces

$$
t+t^{\sigma} \varepsilon=f(x, x) .
$$

However we can replace $t$ with $t+r$ in (23), for any $r \in R$. By comparing the new equation thus obtained with (23) we obtain that $r+r^{\sigma} \varepsilon=0$ for any $r \in R$, 
namely $R \subseteq K^{\sigma, \varepsilon}$. Equivalently, $\bar{R} \subseteq K^{\sigma, \varepsilon} / K_{\sigma, \varepsilon}=\bar{K}^{\circ}$, as claimed in (1). As $\bar{R}$ is o-closed by assumption, $\bar{R}$ is a vector subspace of the $K$-vector space $\bar{K}^{\circ}$.

Claims (2) and (3) can be proved in the same way as claim (1) of Proposition 2.9 and Lemma 2.12, by replacing $K_{\sigma, \varepsilon}$ with $R$ in those proofs.

Note that $f(x, x) \in K_{\sigma,-\varepsilon}$ for any $x \in V$ because $f$ is trace-valued. If $\operatorname{char}(K)=2$ then $\varepsilon=-\varepsilon$. In this case $f(x, x) \in K_{\sigma, \varepsilon} \subseteq R$ for any $x \in V$.

Corollary 3.4. Let $(\sigma, \varepsilon)$ be of trace type and $\bar{R} \neq \bar{K}$. Then $\bar{R}=\{\overline{0}\}$, whence $q$ is pseudo-quadratic.

Proof. By claim (2) of Lemma 2.2, the pairs $(\sigma, \varepsilon)$ is of trace type if and only if $\bar{K}^{\circ}=\{\overline{0}\}$. Moreover, by claim (1) of Theorem 3.3, either $\bar{R}=\bar{K}$ or $\bar{R} \subseteq \bar{K}^{\circ}$. Therefore, if $\bar{R} \subset \bar{K}$ and $\bar{K}^{\circ}=\{\overline{0}\}$ then $\bar{R}=\{\overline{0}\}$.

A generalized pseudo-quadratic form $q: V \rightarrow \bar{K} / \bar{R}$ is said to be trivial if $q(x)=0_{\bar{R}}$ for every $x \in V$.

Proposition 3.5. The form $q$ is trivial if and only if one of the following holds:

(1) $\bar{R}=\bar{K}$.

(2) We have $\bar{R} \neq \bar{K}$ but the sesquilinearization of $q$ is trivial and there exists a basis $\left(e_{i}\right)_{i \in I}$ of $V$ such that $q\left(e_{i}\right)=0_{\bar{R}}$ for every $i \in I$.

Proof. Clearly, if $\bar{R}=\bar{K}$ then $q$ is trivial. Assume that $\bar{R} \subset \bar{K}$. Then $q$ admits a unique sesquilinearization $f$, by Lemma 3.2. Suppose that nevertheless $q$ is trivial. Then $f(x, y) \in R$ for any $x, y \in V$. Accordingly,

$$
\lambda^{\sigma} f(x, y) \mu \in R \text { for any choice of } \lambda, \mu \in K \text { and } x, y \in V .
$$

If $f(x, y) \neq 0$ for a pair $(x, y)$, then (24) forces $R=K$, contrary to the assumptions made on $\bar{R}$. It follows that $f$ is the trivial form.

Conversely, let $f$ be trivial and $q\left(e_{i}\right)=0_{\bar{R}}$ for every $i \in I$. Then the form $g$ defined as in (17) but with $g_{i}=0$ for every $i \in I$, is trivial. However $g$ is a facilitating form of $q$. Hence $q$ is trivial as well.

\subsection{The polar space $S_{q}$}

For the rest of this section we assume that $q$ is non-trivial. In particular, $\bar{R} \neq \bar{K}$. As above, $f$ stands for the sesquilinearization of $q$. The symbol $R$ is given the meaning stated in (20). 
As in the case of pseudo-quadratic forms, we say that a vector $x \in V$ is singular for $q$ (also $q$-singular) if $q(x)=0_{\bar{R}}$. A subspace $X$ of $V$ is totally singular for $q$ (also totally $q$-singular) if $q(x)=0_{\bar{R}}$ for every $x \in X$. Clearly, if $q(x)=0_{\bar{R}}$ for a vector $x \in V$ then $q(x \lambda)=0_{\bar{R}}$ for any $\lambda \in K$. We say that a point $[x]$ of $\mathrm{PG}(V)$ is singular for $q$ (also $q$-singular) if $x$ is $q$-singular. A subspace of $\mathrm{PG}(V)$ is said to be totally singular for $q$ (totally $q$-singular) if all of its points are $q$-singular.

Let $P_{q}$ be the set of $q$-singular points of $\mathrm{PG}(V)$. By claim (2) of Theorem 3.3, if a point of $\mathrm{PG}(V)$ is $q$-singular then it is $f$-isotropic. In short, $P_{q} \subseteq P_{f}$. The following can be proved in the same way as claim (2) of Proposition 2.9, but for replacing $K_{\sigma, \varepsilon}$ with $R$.

Proposition 3.6. A line $[x, y]$ of $\mathrm{PG}(V)$ is totally $q$-singular if and only if $q(x)=$ $q(y)=0_{\bar{R}}$ and $f(x, y)=0$.

Proposition 3.6 immediately implies the following:

Corollary 3.7. A subspace $\left[x_{1}, x_{2}, \ldots, x_{k}\right]$ of $\mathrm{PG}(V)$ is totally $q$-singular if and only if it is totally isotropic for $f$ and $q\left(x_{1}\right)=q\left(x_{2}\right)=\cdots=q\left(x_{k}\right)=0_{\bar{R}}$.

By Corollary 3.4, if $(\sigma, \varepsilon)$ is of trace type then the form $q$ is pseudo-quadratic. By this remark combined with Proposition 2.13 we immediately obtain the following:

Corollary 3.8. Let $(\sigma, \varepsilon)$ be of trace type. Then a subspace of $\mathrm{PG}(V)$ is totally $q$-singular if and only it is totally $f$-isotropic.

Assuming that $P_{q} \neq \emptyset$, let $L_{q}$ be the set of totally $q$-singular lines of $\mathrm{PG}(V)$ and put $S_{q}:=\left(P_{q}, L_{q}\right)$. In view of Proposition 3.7, the point-line geometry $S_{q}$ is a subspace of the polar space $S_{f}=\left(P_{f}, L_{f}\right)$ associated to $f$. Hence $S_{q}$ is itself a polar space. Its radical is a possibly empty subspace of $[\operatorname{Rad}(f)]$, equal to $P_{q} \cap[\operatorname{Rad}(f)]$. If $(\sigma, \varepsilon)$ is of trace type then $S_{q}=S_{f}$, by Corollary 3.8.

We call $S_{q}$ the polar space associated to $q$. The $q$-singular vectors of $\operatorname{Rad}(f)$ form a subspace of $\operatorname{Rad}(f)$, henceforth called the radical of $q$ and denoted by the symbol $\operatorname{Rad}(q)$. We say that $q$ is singular (also degenerate) if $\operatorname{Rad}(q) \neq\{0\}$, namely $S_{q}$ is degenerate. In any case, we call $\operatorname{Rad}(f)$ the defect of $q$.

Let $q_{\mid \operatorname{Rad}(f)}$ be the mapping induced by $q$ on $\operatorname{Rad}(f)$. Clearly $q_{\mid \operatorname{Rad}(f)}$ is additive. This fact and claim (3) of Theorem 3.3 imply the following:

Proposition 3.9. The mapping $q_{\mid \operatorname{Rad}(f)}$ is a homomorphism of $K$-vector spaces from $\operatorname{Rad}(f)$ to $\bar{K}^{\circ} / \bar{R}$ and $\operatorname{Rad}(q)$ is the kernel of this homomorphism.

Consequently, the quotient space $\operatorname{Rad}(f) / \operatorname{Rad}(q)$ is isomorphic to the image $\operatorname{Im}\left(q_{\mid \operatorname{Rad}(f)}\right)$ of $q_{\mid \operatorname{Rad}(f)}$ and the latter is a vector subspace of $\bar{K}^{\circ} / \bar{R}$. 
Finally, the same argument used to prove Proposition 2.11 but with $R$ in the role of $K_{\sigma, \varepsilon}$, yields the following:

Proposition 3.10. Either $P_{q}$ is a subspace of $\mathrm{PG}(V)$ or it spans $\mathrm{PG}(V)$.

\subsection{A facilitating form}

We keep the hypotheses and the notation of the previous subsection. In particular, $\bar{R} \neq \bar{K}, f$ is the sesquilinearization of $q$ and $P_{q}$ is the set of $q$-singular points of $\mathrm{PG}(V)$. We also assume that $P_{q}$ spans $\mathrm{PG}(V)$. Hence $V$ admits a basis formed by $q$-singular vectors. We call such a basis a $q$-singular basis.

Let $E=\left(e_{i}\right)_{i \in I}$ be a $q$-singular basis of $V$. Given a total ordering $<$ on the set $I$ of indices, let $g_{E}: V \times V \rightarrow K$ be the $\sigma$-sequilinear form defined as follows:

$$
g_{E}\left(\sum_{i} e_{i} \lambda_{i}, \sum_{j} e_{j} \mu_{j}\right):=\sum_{i<j} \lambda^{\sigma} f\left(e_{i}, e_{j}\right) \mu_{j} .
$$

Since $q\left(e_{i}\right)=0_{\bar{R}}$ for every $i \in I$, the form $g_{E}$ is a facilitating form for $q$, namely

$$
q(x)=\overline{g_{E}(x, x)}+\bar{R}=\sum_{i<j} \overline{\lambda_{i}^{\sigma} f\left(e_{i}, e_{j}\right) \lambda_{j}}+\bar{R}
$$

for every vector $x=\sum_{i \in I} e_{i} \lambda_{i}$ of $V$. Clearly, the coset $\overline{g_{E}(x, x)}+\bar{R}$ does not depend on the choice of the $q$-singular basis $E$ but the scalar $g_{E}(x, x)$ obviously depends on that choice. The value $\overline{g_{E}(x, x)}$ also depends on it, to some extent. In order to make this remark less vague, we need a few additional definitions. Let $E=\left(e_{i}\right)_{i \in I}$ and $E^{\prime}=\left(e_{i}^{\prime}\right)_{i \in I}$ be two ordered $q$-singular bases of $V$. Let $\bar{R}_{E, E^{\prime}}$ be the o-closed subgroup of $\bar{K}$ spanned by the family $\left\{\overline{g_{E^{\prime}}\left(e_{i}, e_{i}\right)}\right\}_{i \in I}$ and let $\delta_{E, E^{\prime}}: V \in \bar{K}$ be the mapping defined as follows:

$$
\delta_{E, E^{\prime}}(x):=\overline{g_{E}(x, x)}-\overline{g_{E^{\prime}}(x, x)} .
$$

Clearly, $\delta_{E, E^{\prime}}(x)+\bar{R}=q(x)-q(x)=0_{\bar{R}}$. Therefore $\delta_{E, E^{\prime}}(V) \subseteq \bar{R}$. Recall that $\bar{R}$ is a vector subspace of $\bar{K}^{\circ}$, as we know from claim (1) of Theorem 3.3.

Lemma 3.11. The group $\bar{R}_{E, E^{\prime}}$, equipped with the scalar multiplication $\circ$, is a vector subspace of $\bar{R}$ and $\delta_{E, E^{\prime}}$ is a surjective linear map from $V$ to $\bar{R}_{E, E^{\prime}}$. Moreover $\delta_{E^{\prime}, E}=-\delta_{E . E^{\prime}}$ and $\bar{R}_{E, E^{\prime}}=\bar{R}_{E^{\prime}, E}$.

Proof. For $x \in V$ let $x=\sum_{i} e_{i} \lambda_{i}=\sum_{i} e_{i}^{\prime} \lambda_{i}^{\prime}$. Then

$$
\left\{\begin{array}{c}
g_{E}(x, x)=\sum_{i<j} \lambda_{i}^{\sigma} f\left(e_{i}, e_{j}\right) \lambda_{j}, \\
g_{E^{\prime}}(x, x)=\sum_{i<j}\left(\lambda_{i}^{\prime}\right)^{\sigma} f\left(e_{i}^{\prime}, e_{j}^{\prime}\right) \lambda_{j}^{\prime} .
\end{array}\right.
$$


Moreover, there exist scalars $\alpha_{i j}(i, j \in I)$ such that

$$
e_{k}=\sum_{i} e_{i}^{\prime} \alpha_{i k} \text { for all } k \in I .
$$

Hence

$$
\lambda_{k}^{\prime}=\sum_{i} \alpha_{k i} \lambda_{i} \text { for all } k \in I .
$$

Substituting (27) in the first equality of (26) and (28) in the second one we get

$$
\left\{\begin{array}{c}
g_{E}(x, x)=\sum_{i<j} \sum_{k, h} \lambda_{i}^{\sigma} \alpha_{k, i}^{\sigma} f\left(e_{k}^{\prime}, e_{h}^{\prime}\right) \alpha_{h, j} \lambda_{j}, \\
g_{E^{\prime}}(x, x)=\sum_{i<j} \sum_{k, h} \lambda_{k}^{\sigma} \alpha_{i, k}^{\sigma} f\left(e_{i}^{\prime}, e_{j}^{\prime}\right) \alpha_{j, h} \lambda_{h} .
\end{array}\right.
$$

By changing indices in the second equation of (29), we can rewrite the two equations (29) as follows:

$$
\left\{\begin{aligned}
g_{E}(x, x) & =\sum_{i, j, k, h ; i<j} \lambda_{i}^{\sigma} \alpha_{k, i}^{\sigma} f\left(e_{k}^{\prime}, e_{h}^{\prime}\right) \alpha_{h, j} \lambda_{j}, \\
g_{E^{\prime}}(x, x) & =\sum_{i, j, k, h ; k<h} \lambda_{i}^{\sigma} \alpha_{k, i}^{\sigma} f\left(e_{k}^{\prime}, e_{h}^{\prime}\right) \alpha_{h, j} \lambda_{j} .
\end{aligned}\right.
$$

Recalling that $f\left(e_{h}^{\prime}, e_{k}^{\prime}\right)=f\left(e_{k}^{\prime}, e_{h}^{\prime}\right)^{\sigma} \varepsilon$, that

$$
\begin{aligned}
\lambda_{i}^{\sigma} \alpha_{k, i}^{\sigma} f\left(e_{k}^{\prime}, e_{h}^{\prime}\right) \alpha_{h, j} \lambda_{j}-\lambda_{j}^{\sigma} \alpha_{h, j}^{\sigma} f\left(e_{k}^{\prime}, e_{h}^{\prime}\right)^{\sigma} \varepsilon \alpha_{k, i} \lambda_{i} \\
=\left(\lambda_{i}^{\sigma} \alpha_{k, i}^{\sigma} f\left(e_{k}^{\prime}, e_{h}^{\prime}\right) \alpha_{h, j} \lambda_{j}\right)-\left(\lambda_{i}^{\sigma} \alpha_{k, i}^{\sigma} f\left(e_{k}^{\prime}, e_{h}^{\prime}\right) \alpha_{h, j} \lambda_{j}\right)^{\sigma} \varepsilon \in K_{\sigma, \varepsilon},
\end{aligned}
$$

and $f\left(e_{k}^{\prime}, e_{k}^{\prime}\right)=0$ (by (2) of Theorem 3.3 and since $q\left(e_{k}^{\prime}\right)=0 \bar{R}$ by assumption), we can rewrite the two equalities (30) as follows:

$$
\begin{array}{r}
g_{E}(x, x)=\sum_{i<j, k<h} \lambda_{i}^{\sigma} \alpha_{k, i}^{\sigma}\left(f\left(e_{k}^{\prime}, e_{h}^{\prime}\right)+f\left(e_{k}^{\prime}, e_{h}^{\prime}\right)^{\sigma} \varepsilon\right) \alpha_{h, j} \lambda_{j}, \\
g_{E^{\prime}}(x, x)+K_{\sigma, \varepsilon}=\sum_{i<j, k<h} \lambda_{i}^{\sigma} \alpha_{k, i}^{\sigma}\left(f\left(e_{k}^{\prime}, e_{h}^{\prime}\right)+f\left(e_{k}^{\prime}, e_{h}^{\prime}\right)^{\sigma} \varepsilon\right) \alpha_{h, j} \lambda_{j} \\
+\sum_{k, h, i ; k<h} \lambda_{i}^{\sigma} \alpha_{k, i}^{\sigma} f\left(e_{k}^{\prime}, e_{h}^{\prime}\right) \alpha_{h, i} \lambda_{i}+K_{\sigma, \varepsilon} .
\end{array}
$$

Consequently,

$$
\overline{g_{E}(x, x)}-\overline{g_{E^{\prime}}(x, x)}=-\sum_{k, h, i ; k<h} \overline{\alpha_{k, i}^{\sigma} f\left(e_{k}^{\prime}, e_{h}^{\prime}\right) \alpha_{h, i}} \circ \lambda_{i} .
$$

However $\sum_{k<h} \alpha_{k, i}^{\sigma} f\left(e_{k}^{\prime}, e_{k}^{\prime}\right) \alpha_{h, i}=g_{E^{\prime}}\left(\sum_{k} e_{k}^{\prime} \alpha_{k, i}, \sum_{k} e_{k}^{\prime} \alpha_{k, i}\right)=g_{E^{\prime}}\left(e_{i}, e_{i}\right)$ by (27) and the definition of $g_{E^{\prime}}$. Substituing in (31) we obtain:

$$
\overline{g_{E}(x, x)}-\overline{g_{E^{\prime}}(x, x)}=-\sum_{i} \overline{g_{E^{\prime}}\left(e_{i}, e_{i}\right)} \circ \lambda_{i} .
$$

According to (32), we have $\bar{R}_{E, E^{\prime}}=\delta_{E, E^{\prime}}(V)$ ( $\subseteq \bar{R}$, as previously remarked). Therefore $\bar{R}_{E, E^{\prime}}$ is a vector subspace of $\bar{R}$. Equation (32) also shows that $\delta_{E, E^{\prime}}$ is a linear mapping from $V$ to $\bar{R}_{E, E^{\prime}}$. Clearly, $\delta_{E^{\prime}, E}=-\delta_{E, E^{\prime}}$. Whence $\bar{R}_{E, E^{\prime}}=$ $\bar{R}_{E^{\prime}, E}$. 
We call $\delta_{E, E^{\prime}}$ and $\bar{R}_{E, E^{\prime}}$ the difference-map and the difference-space relative to the pair $\left(E, E^{\prime}\right)$ of $q$-singular bases.

Remark 3.12. Only $q$-singular bases are considered in Lemma 3.11, but the statement of Lemma 3.11 holds for any pair of bases formed by $f$-isotropic vectors, except that in this more general setting no closed subgroup $\bar{R}$ is given in advance. Instead of $\bar{R}$ we must consider the closed subgroups $\bar{R}_{E}$ and $\bar{R}_{E^{\prime}}$ of $\bar{K}$ generated by the sets $\left\{\overline{g_{E}(x, x)}\right\}_{[x] \in P_{f}}$ and $\left\{\overline{g_{E^{\prime}}(x, x)}\right\}_{[x] \in P_{f}}$ respectively. The proof of Lemma 3.11 shows that $\delta_{E, E^{\prime}}(V)=\bar{R}_{E, E^{\prime}} \subseteq \bar{R}_{E^{\prime}}$, whence $\bar{R}_{E} \subseteq \bar{R}_{E^{\prime}}$. By symmetry, $\bar{R}_{E} \supseteq \bar{R}_{E^{\prime}}$. Finally $\bar{R}_{E}=\bar{R}_{E^{\prime}}$.

For every $x \in V$, put $\gamma_{E}(x):=\overline{g_{E}(x, x)}$ and $\gamma_{E^{\prime}}(x):=\overline{g_{E^{\prime}}(x, x)}$. Then both $\gamma_{E}$ and $\gamma_{E^{\prime}}$ are pseudo-quadratic forms. By Lemma 2.12, the group $\bar{R}_{E}=\bar{R}_{E^{\prime}}$ is a vector subspace of $\bar{K}^{\circ}$.

\subsection{Isomorphisms and weak isomorphisms}

Given two generalized $(\sigma, \varepsilon)$-quadratic forms $q: V \rightarrow \bar{K} / \bar{R}$ and $q^{\prime}: V^{\prime} \rightarrow \bar{K} / \bar{R}$ with the same co-defect $\bar{R}$, we say that $q$ and $q^{\prime}$ are isomorphic if there exists a bijective linear mapping $\alpha: V \rightarrow V^{\prime}$ such that $q^{\prime}(\alpha(x))=q(x)$ for every $x \in V$. A broader notion of isomorphism can also be considered, but before to state it we need a few preliminary remarks on automorphisms of $K$.

We say that an automorphism $\rho$ of $K$ stabilizes a given admissible pair $(\sigma, \varepsilon)$ if $\rho \sigma=\sigma \rho$ and $\varepsilon^{\rho}=\varepsilon$. Let $\rho \in \operatorname{Aut}(K)$ stabilize $(\sigma, \varepsilon)$. Then $\rho$ stabilizes both $K_{\sigma, \varepsilon}$ and $K^{\sigma, \varepsilon}$. Thus $\rho$ induces on the group $\bar{K}=K / K_{\sigma, \varepsilon}$ an automorphism $\bar{\rho}$ stabilizing $\bar{K}^{\circ}=K^{\sigma, \varepsilon} / K_{\sigma, \varepsilon}$. Moreover, $(\bar{t} \circ \lambda)^{\bar{\rho}}=\bar{t}^{\bar{\rho}} \circ \lambda^{\rho}$ for every element $\bar{t} \in \bar{K}$ and every scalar $\lambda \in K$. Hence the automorphism of $\bar{K}^{\circ}$ induced by $\bar{\rho}$ is a bijective $\rho$-semi-linear mapping of the $K$-vector space $\bar{K}^{\circ}$.

Given a o-closed subgroup $\bar{R}$ of $\bar{K}$, let $\bar{R}^{\bar{\rho}}$ be the image of $\bar{R}$ by $\bar{\rho}$. Then $\bar{R}^{\bar{\rho}}$ is o-closed and $\bar{\rho}$ induces an isomorphism from $\bar{K} / \bar{R}$ to $\bar{K} / \bar{R}^{\bar{\rho}}$. Clearly, for every element $\bar{t}+\bar{R}$ of $\bar{K} / \bar{R}$ and every $\lambda \in K$ we have

$$
((\bar{t}+\bar{R}) \circ \lambda)^{\bar{\rho}}=\left(\bar{t}^{\bar{\rho}}+\bar{R}^{\bar{\rho}}\right) \circ \lambda^{\rho}=(\bar{t}+\bar{R})^{\bar{\rho}} \circ \lambda^{\rho} .
$$

We can now weaken our previous definition of isomorphism. Let $\bar{R}$ and $\bar{R}^{\prime}$ be two o-closed subgroups of $K$. We say that two generalized $(\sigma, \varepsilon)$-quadratic forms $q: V \rightarrow \bar{K} / \bar{R}$ and $q^{\prime}: V^{\prime} \rightarrow \bar{K} / \bar{R}^{\prime}$ are weakly isomorphic if there exists an automorphism $\rho$ of $K$ stabilizing $(\sigma, \varepsilon)$ and such that $\bar{R}^{\bar{\rho}}=\bar{R}^{\prime}$ and a $\rho$-semilinear map $\alpha: V \rightarrow V^{\prime}$ such that $q^{\prime}(\alpha(x))=q(x)^{\bar{\rho}}$ for every $x \in V$. 


\subsection{Proportionality}

For $i=1,2$ let $\left(\sigma_{i}, \varepsilon_{i}\right)$ be an admissible pair of $K$ and $\bar{R}_{i}$ a $\circ_{\sigma_{i}}$-closed subgroup of $\bar{K}^{\sigma_{i}, \varepsilon_{i}}=K / K_{\sigma_{i}, \varepsilon_{i}}$ (notation as in Subsection 2.1.4). Let $q_{i}: V \rightarrow \bar{K}^{\sigma_{i}, \varepsilon_{i}} / \bar{R}_{i}$ be a non-trivial generalized $\left(\sigma_{i}, \varepsilon_{i}\right)$-quadratic form and let $f_{i}$ be its sesquilinearization. We say that $q_{1}$ and $q_{2}$ are proportional if there exists a scalar $\kappa \in K-\{0\}$ such that $\left(\sigma_{2}, \varepsilon_{2}\right)=\kappa \cdot\left(\sigma_{1}, \varepsilon_{1}\right), \bar{R}_{2}=\kappa \bar{R}_{1}$ and $q_{2}(x)=\kappa q_{1}(x)$ for every $x \in V$. If this is the case then we write $q_{2}=\kappa q_{1}$. Clearly, if $q_{2}=\kappa q_{1}$ then $f_{2}=\kappa f_{1}$ and $S_{q_{1}}=S_{q_{2}}$.

Theorem 3.13. Let $q_{1}: V \rightarrow \bar{K}^{\sigma_{1}, \varepsilon_{1}} / \bar{R}_{1}$ and $q_{2}: V \rightarrow \bar{K}^{\sigma_{2}, \varepsilon_{2}} / \bar{R}_{2}$ be generalized pseudo-quadratic forms such that $S_{q_{1}}=S_{q_{2}}$. Assume that the polar space $S:=$ $S_{q_{1}}=S_{q_{2}}$ has non-degenerate rank at least 2 . Then $q_{1}$ and $q_{2}$ are proportional.

Proof. By the same argument used in the proof of Proposition 2.14 we obtain that $f_{1}$ and $f_{2}$ are proportional. Thus, modulo replacing $q_{1}$ with $\kappa q_{1}$ for a suitable $\kappa \in K-\{0\}$ me may assume that $f_{1}=f_{2}=f$, say. Hence $\left(\sigma_{1}, \varepsilon_{1}\right)=\left(\sigma_{2}, \varepsilon_{2}\right)$ and $\bar{K}^{\sigma_{1}, \varepsilon_{1}}=\bar{K}^{\sigma_{2}, \varepsilon_{2}}=: \bar{K}$. We must prove that we also have $q_{1}=q_{2}$. As $f_{1}=f_{2}=f$, we can choose the same facilitating form $g$ for $q_{1}$ and $q_{2}$, defining it as in (25) of Subsection 3.4. So, for every $x \in V$, we can choose the same representative $\bar{t}_{x} \in \bar{K}$ for both $q_{1}(x)$ and $q_{2}(x)$. In order to prove that $q_{1}=q_{2}$ we must only show that $\bar{R}_{1}=\bar{R}_{2}$.

Let $\bar{r} \in \bar{R}_{1}$. Let $a$ and $b$ be two vectors such that $f(a, b)=1$ and $[a],[b] \in S$ ( $=S_{q_{1}}=S_{q_{2}}$ ). Such a pair of vectors exists in view of the hypotheses made on $S$. Let $r \in K$ be such that $\bar{r} \in \bar{R}_{1}$. Then $q_{1}(a+b r)=\bar{r}+\bar{R}_{1}=\bar{R}_{1}$. Hence $[a+b r] \in S$. On the other hand, $q_{2}(a+b r)=\bar{r}+\bar{R}_{2}$. As $[a+b r] \in S=S_{q_{2}}$, the vector $a+b r$ is $q_{2}$-singular, hence $\bar{r} \in \bar{R}_{2}$. It follows that $\bar{R}_{1} \subseteq \bar{R}_{2}$. By symmetry, $\bar{R}_{2} \subseteq \bar{R}_{1}$. Finally, $\bar{R}_{1}=\bar{R}_{2}$.

\section{Quotients and covers}

In this section $q: V \rightarrow \bar{K} / \bar{R}$ is a given non-trivial generalized $(\sigma, \varepsilon)$-quadratic form, $f: V \times V \rightarrow K$ is its sesquilinearization and $S_{q}=\left(P_{q}, L_{q}\right)$ is the polar space associated to $q$. As $q$ is non-trivial, the form $f$ is non-trivial as well, by Proposition 3.5. Moreover, $\bar{R}$ is a vector subspace of $\bar{K}^{\circ}$, by Theorem 3.3, (1).

We assume that $P_{q}$ is not totally singular. Hence it spans $\mathrm{PG}(V)$ (Proposition 3.10). Therefore the inclusion mapping $e_{q}: S_{q} \rightarrow \mathrm{PG}(V)$ is an embedding of $S_{q}$ in $\mathrm{PG}(V)$. Recall that $[\operatorname{Rad}(q)]=[\operatorname{Rad}(f)] \cap P_{q}$ is the radical of $S_{q}$. 


\subsection{Quotients}

According to the definitions stated in Subsection 1.3.3, a subspace $U$ of $V$ defines a quotient of the embedding $e_{q}: S_{q} \rightarrow \mathrm{PG}(V)$ precisely when $[U] \cap P_{q}=\emptyset$ and $[U] \cap[a, b]=\emptyset$ for any two distinct points $[a],[b] \in P_{q}$.

Proposition 4.1. A subspace $U$ of $V$ defines a quotient of the embedding $e_{q}$ if and only if $U \subseteq \operatorname{Rad}(f)$ and $U \cap \operatorname{Rad}(q)=0$.

Proof. As said in Subsection 1.3.3, a subspace $U$ of $V$ defines a quotient of $e_{q}$ if and only if $[U] \cap P_{q}=\emptyset$ and every line of $\mathrm{PG}(V)$ meeting $[U]$ non-trivially meets $P_{q}$ in at most one point. So, in order to prove Proposition 4.1 we only must prove that a point $[v]$ of $\mathrm{PG}(V)-P_{q}$ belongs to $[\operatorname{Rad}(f)]$ if and only if every projective line through $[v]$ meets $P_{q}$ in at most one point.

Given a point $[v] \notin P_{q}$, assume firstly that every projective line through $[v]$ meets $P_{q}$ in at most one point. Let $[a] \in P_{q}$. Then $q(a)=0_{\bar{R}}$. Hence $q(a \lambda+v)=$ $q(v)+\left(\overline{\lambda^{\sigma} f(a, v)}+\bar{R}\right)$ for any $\lambda \in K$. It follows that if $f(a, v) \neq 0$ then a scalar $\lambda \in K$ exists such that $q(a \lambda+v)=0_{\bar{R}}$. If this is the case then $[a, v]$ meets $P_{q}$ in at least two points, namely $[a]$ and $[a \lambda+v]$, a contradiction with the hypotheses made on $[v]$. Therefore $f(a, v)=0$. As this holds for any $[a] \in P_{q}$, it follows that $P_{q} \subseteq\left[v^{\perp}\right]$. However $P_{q}$ spans $\operatorname{PG}(V)$, by assumption. Hence $V=v^{\perp}$, namely $v \in \operatorname{Rad}(f)$.

Conversely, let $v \in \operatorname{Rad}(f)$. Let $[a] \in P_{q}$. Then $q(a)=0_{\bar{R}}$ and $f(a, v)=0$ while $q(v) \neq 0_{\bar{R}}$ as $[v] \notin P_{q}$ by assumption. Hence $q(a \lambda+v)=q(v) \neq 0_{\bar{R}}$ for any $\lambda \in K$. This shows that $[a, v] \cap P_{q}=\{[a]\}$. Therefore every projective line through $[v]$ meets $P_{q}$ in at most one point.

The next corollary immediately follows from Proposition 4.1.

Corollary 4.2. If $\operatorname{Rad}(q)=\operatorname{Rad}(f)$ then the embedding $e_{q}$ does not admit any proper quotient.

For the rest of this subsection we assume that $\operatorname{Rad}(q) \neq \operatorname{Rad}(f)$. Hence $S_{q}$ is a proper subspace of $S_{f}$. Consequently, $(\sigma, \varepsilon)$ is not of trace type, by Corollary 3.8. In particular, $\operatorname{char}(K)=2$.

Let $U$ be a subspace of $\operatorname{Rad}(f)$ with $U \cap \operatorname{Rad}(q)=0$. By Proposition 3.9, the restriction of $q$ to $U$ is an injective linear mapping from $U$ to the $K$-vector space $\bar{K}^{\circ} / \bar{R}$. Hence the image $q(U)$ of $U$ by $q$ is a vector subspace of $\bar{K}^{\circ} / \bar{R}$. Therefore there exists a unique subspace $\bar{R}_{U}$ of $\bar{K}^{\circ}$ containing $\bar{R}$ and such that $\bar{R}_{U} / \bar{R}=q(U)$. Let $q_{U}: V / U \rightarrow \bar{K} / \bar{R}_{U}$ be the mapping defined as follows:

$$
q_{U}(x+U)=\bar{t}+\bar{R}_{U} \text { for an element } t \in K \text { such that } \bar{t}+\bar{R}=q(x) .
$$


Lemma 4.3. The mapping $q_{U}$ is well defined.

Proof. The coset $\bar{t}+\bar{R}_{U}$ does not depend on the choice of the representative $\bar{t}$ of $q(x)$. It remains to prove that it neither depends on the choice of the vector $x$ in the coset $x+U$.

Given $u \in U$, let $x^{\prime}=x+u$ and let $\bar{t}^{\prime}$ be a representative of $q\left(x^{\prime}\right)$. Then $q\left(x^{\prime}\right)=q(x+u)=q(x)+q(u)+(f(x, u)+\bar{R})=q(x)+q(u)$ because $u \in U \subseteq$ $\operatorname{Rad}(f)$. However $q(u) \in \bar{R}_{U} / \bar{R}$ by definition of $\bar{R}_{U}$. Therefore $\bar{t}-\bar{t}^{\prime} \in \bar{R}_{U}$, namely $\bar{t}+\bar{R}_{U}=\bar{t}^{\prime}+\bar{R}_{U}$.

The sesquilinearization $f$ of $q$ induces a trace-valued $(\sigma, \varepsilon)$-sesquilinear form $f_{U}$ on $V / U$. Explicitly,

$$
f_{U}(x+U, y+U):=f(x, y) .
$$

This definition is consistent. Indeed, since $U \subseteq \operatorname{Rad}(f)$, we have $f(x+u, y+v)=$ $f(x, y)$ for any choice of $u, v \in U$. It is clear that, since $f$ is trace-valued and non-trivial, $f_{U}$ is trace-valued and non-trivial as well. The proof of the following lemma is straightforward.

Lemma 4.4. The mapping $q_{U}$ is a generalized $(\sigma, \varepsilon)$-quadratic form. The form $f_{U}$ induced by $f$ on $V / U$ is a sesquilinearization of $q_{U}$.

As $f_{U}$ is non-trivial, the form $q_{U}$ is non-trivial if and only if $\bar{R}_{U} \neq \bar{K}$, by Proposition 3.5. If this is the case then $f_{U}$ is the unique sesquilinearization of $q_{U}$, by Lemma 3.2. Finally, Lemma 4.4 and claim (1) of Theorem 3.3 imply the following:

Corollary 4.5. Let $q_{U}$ be non-trivial. Then $\bar{R}_{U} \subseteq \bar{K}^{\circ}$.

We call $q_{U}$ the quotient of $q$ by $U$. According to the notation of Subsection 3.3, when $q_{U}$ is non-trivial $P_{q_{U}}$ and $L_{q_{U}}$ are the set of $q_{U}$-singular points and the set of totally $q_{U}$-singular lines of $\mathrm{PG}(V / U)$ respectively and $S_{q_{U}}=\left(P_{q_{U}}, L_{q_{U}}\right)$ is the polar space associated to $q_{U}$ in $\mathrm{PG}(V / U)$.

Theorem 4.6. Let $\pi_{U}: V \rightarrow V / U$ be the projection of $V$ onto $V / U$.

(1) Let $q_{U}$ be non-trivial. Then $\pi_{U}$ induces an isomorphism from $S_{q}$ to $S_{q_{U}}$.

(2) Let $q_{U}$ be trivial. Then both forms $f$ and $f_{U}$ are alternating and $\pi_{U}$ induces an isomorphism from $S_{q}$ to the polar space $S_{f_{U}}$ associated to $f_{U}$.

Proof. As $U$ defines a quotient of $S_{q}$, every coset $x+U$ of $U$ in $V$ contains at most one $q$-singular vector. Therefore $\pi_{U}$ induces and injective mapping on $P_{q}$. We firstly prove the following: 
(*) For every non-zero vector $x \in V, q_{U}(x+U)=0_{\bar{R}_{U}}$ if and only if $x+u$ is $q$-singular for some $u \in U$.

The coset $x+U$ contains a $q$-singular vector if and only if $q(x+u)=0_{\bar{R}}$ for some vector $u \in U$, namely $q(x)+q(u)=0_{\bar{R}}$. (Recall that $f(x, u)=0$ since $U \subseteq \operatorname{Rad}(f))$. If this is the case then $q(x) \in \bar{R}_{U} / \bar{R}$, namely $q_{U}(x+U)=0_{\bar{R}_{U}}$. Conversely, let $q_{U}(x+U)=0_{\bar{R}_{U}}$. Then there exists an element $\bar{t} \in \bar{R}_{U}$ such that $q(x)=\bar{t}+\bar{R}$. By definition of $\bar{R}_{U}$, we have $\bar{t}+\bar{R}=q(u)$ for some $u \in U$. Hence $q(x-u)=0_{\bar{R}}$, namely $x-u$ is $q$-singular. Claim $(*)$ is proved.

Let $q_{U}$ be non-trivial. By $(*)$, the projection $\pi_{U}$ induces a bijection from $P_{q}$ to $P_{q_{U}}$. Two $q_{U}$-singular points $[x+U]$ and $[y+U]$ of $\mathrm{PG}(V / U)$ are collinear in $S_{q_{U}}$ if and only if $f_{U}(x+U, y+U)=0$. By the definition of $f_{U}$, this condition is equivalent to $f(x, y)=0$, which in its turn characterizes the collinearity of $[x]$ and $[y]$. Claim (1) of the theorem is proved.

Let $q_{U}$ be trivial. Then $(*)$ shows that $\pi_{U}$ induces a bijection from $P_{q}$ to the set of points of $\mathrm{PG}(V / U)$. In other words, every coset $x+U$ of $U$ other than $U$ contains exactly one $q$-singular vector. We may assume that in a symbol as $x+U$ the letter $x$ stands for the unique $q$-singular vector of $x+U$. With this convention, $f_{U}(x+U, x+U)=f(x, x)$ (by definition of $f_{U}$ ) and $f(x, x)=0$ because $x$ is $q$-singular, whence $f$-isotropic. It follows that $f_{U}(x+U, x+U)=0$ for every coset $x+U$. Thus, $f_{U}$ is alternating. Moreover, for any vector $x \in V$ we have $f(x, x)=f_{U}(x+U, x+U)$ by definition of $f_{U}$ and $f_{U}(x+U, x+U)=0$ since $f_{U}$ is alternating. Hence $f(x, x)=0$ for every $x \in V$, namely $f$ is alternating as well. Turning to $S_{q}$, two points $[x],[y] \in S_{q}$ are collinear in $S_{q}$ if and only if $f(x, y)=0$, equivalently $f_{U}(x+U, y+U)=0$, namely $x+U$ and $y+U$ represent collinear points of $S_{f_{U}}$. Therefore $\pi_{U}$ maps $S_{q}$ isomorphically onto $S_{f_{U}}$, as claimed in (2).

\subsection{Covers}

Let $\bar{S} \oplus \bar{T}=\bar{R}$ be a direct sum decomposition of the $K$-vector space $\bar{R}$. Put $V^{\bar{S}}:=V \oplus \bar{S}$ (direct sum of $K$-vector spaces). Define $f^{\bar{S}}: V^{\bar{S}} \times V^{\bar{S}} \rightarrow K$ as follows:

$$
f^{\bar{S}}(x+\bar{r}, y+\bar{s})=f(x, y) \text { for all } x, y \in V \text { and } \bar{r}, \bar{s} \in \bar{S} .
$$

It is easy to see that $f^{\bar{S}}$ is a trace-valued $(\sigma, \varepsilon)$-sesquilinear form with $\operatorname{Rad}\left(f^{\bar{S}}\right)=$ $\operatorname{Rad}(f) \oplus \bar{S}$. Clearly, $f$ is isomorphic to the form induced by $f^{\bar{S}}$ on $V^{\bar{S}} / \bar{S}(\cong V)$.

Let $E=\left(e_{i}\right)_{i \in I}$ be a $q$-singular basis of $V$ and let $g_{E}$ be the facilitating form associated to $E$ (Subsection 3.4, definition (25)). We define a mapping 
$q_{E}^{\bar{S}, \bar{T}}: V^{\bar{S}} \rightarrow \bar{K} / \bar{T}$ as follows:

$$
q_{E}^{\bar{S}, \bar{T}}(x+\bar{r})=\overline{g_{E}(x, x)}+\bar{r}+\bar{T} \text { for any } x \in V \text { and any } \bar{r} \in \bar{S} .
$$

In particular, $q_{E}^{\bar{S}}, \bar{T}(x)=\overline{g_{E}(x, x)}+\bar{T}$ and $q_{E}^{\bar{S}}, \bar{T}(\bar{r})=\bar{r}+\bar{T}$.

Theorem 4.7. The map $q_{E}^{\bar{S}}, \bar{T}$ is a non-trivial generalized $(\sigma, \varepsilon)$-quadratic form and $f^{\bar{S}}$ is its sesquilinearization.

Proof. Let $x=\sum_{i} e_{i} \lambda_{i}$ and $\bar{r} \in \bar{S}$. According to the definition of $q_{E}^{\bar{S}, \bar{T}}$ we have

$$
\begin{aligned}
q_{E}^{\bar{S}, \bar{T}}((x+\bar{r}) \lambda)= & q_{E}^{\bar{S}, \bar{T}}(x \lambda+\bar{r} \circ \lambda)=\sum_{i<j} \overline{\lambda^{\sigma} \lambda_{i}^{\sigma} f\left(e_{i}, e_{j}\right) \lambda_{j} \lambda}+\bar{r} \circ \lambda+\bar{T} \\
& =\left(\sum_{i<j} \overline{\lambda_{i}^{\sigma} f\left(e_{i}, e_{j}\right) \lambda_{j}}\right) \circ \lambda+\bar{r} \circ \lambda+\bar{T}=q_{E}^{\bar{S}, \bar{T}}(x+\bar{r}) \circ \lambda .
\end{aligned}
$$

So, $q_{E}^{\bar{S}, \bar{T}}$ satisfies condition (Q'1). Turning to (Q'2), let $x=\sum_{i} e_{i} \lambda_{i}, y=\sum_{i} e_{i} \mu_{i}$ and $\bar{r}, \bar{s} \in \bar{S}$. Then

$$
\begin{aligned}
q_{E}^{\bar{S}, \bar{T}}((x+\bar{r})+(y+\bar{s}))=q_{E}^{\bar{S}, \bar{T}} & ((x+y)+(\bar{r}+\bar{s})) \\
& =\sum_{i<j} \overline{f\left(e_{i}, e_{j}\right)} \circ\left(\lambda_{j}+\mu_{j}\right)+\bar{r}+\bar{s}+\bar{T} .
\end{aligned}
$$

On the other hand,

$$
\begin{aligned}
& q_{E}^{\bar{S}, \bar{T}}(x+\bar{r})+q_{E}^{\bar{S}, \bar{T}}(y+\bar{s}) \\
& \quad=\sum_{i<j} \overline{f\left(e_{i}, e_{j}\right)} \lambda_{j}+\sum_{i<j} \overline{f\left(e_{i}, e_{j}\right)} \mu_{j}+\bar{r}+\bar{s}+\bar{T} .
\end{aligned}
$$

Moreover,

$$
f^{\bar{S}}(x+\bar{r}, y+\bar{s})=f(x, y)=\sum_{i<j}\left(\lambda_{i}^{\sigma} f\left(e_{i}, e_{j}\right) \mu_{j} .\right.
$$

By (33), (34) and (35) and recalling that

$$
\begin{aligned}
\mu_{i}^{\sigma} f\left(e_{i}, e_{j}\right) \lambda_{j}-\lambda_{j}^{\sigma} f\left(e_{j}, e_{i}\right) \mu_{i}= & \mu_{i}^{\sigma} f\left(e_{i}, e_{j}\right) \lambda_{j}-\lambda_{j}^{\sigma} f\left(e_{i}, e_{j}\right)^{\sigma} \varepsilon \mu_{i} \\
& =\mu_{i}^{\sigma} f\left(e_{i}, e_{j}\right) \lambda_{j}-\left(\mu_{i}^{\sigma} f\left(e_{i}, e_{j}\right) \lambda_{i}\right)^{\sigma} \varepsilon \in K_{\sigma, \varepsilon}
\end{aligned}
$$

we obtain

$$
\begin{aligned}
q_{E}^{\bar{S}, \bar{T}}((x+\bar{r}) & +(y+\bar{s}))-q_{E}^{\bar{S}, \bar{T}}(x+\bar{r})-q_{E}^{\bar{S}, \bar{T}}(y+\bar{s})-(\overline{f(x+\bar{r}, y+\bar{s})}+\bar{T}) \\
& =\sum_{i<j}\left(\overline{\lambda_{j}^{\sigma} f\left(e_{j}, e_{i}\right) \mu_{i}}+\overline{\lambda_{i}^{\sigma} f\left(e_{i}, e_{j}\right) \mu_{j}}-\sum_{i, j} \overline{\lambda^{\sigma} f\left(e_{i}, e_{j}\right) \mu_{j}}+\bar{T}\right. \\
& =\sum_{i} \overline{\lambda_{i}^{\sigma} f\left(e_{i}, e_{i}\right) \mu_{i}}+\bar{T}=\bar{T} .
\end{aligned}
$$

(Recall that $f\left(e_{i}, e_{i}\right)=0$ since $q\left(e_{i}\right)=0_{\bar{R}}$ by assumption.) Finally,

$q_{E}^{\bar{S}, \bar{T}}((x+\bar{r})+(y+\bar{s}))-q_{E}^{\bar{S}, \bar{T}}(x+\bar{r})-q_{E}^{\bar{S}, \bar{T}}(y+\bar{s})-(\overline{f(x+\bar{r}, y+\bar{s})}+\bar{T})=\bar{T}$.

Property (Q'2) is proved. The non-triviality of $q_{E}^{\bar{S}, \bar{T}}$ immediately follows from the fact that $q$ is non-trivial by assumption. 
We say that $q_{E}^{\bar{S}, \bar{T}}$ is the cover of $q$ via $(\bar{S}, \bar{T})$ based at $E$ (a cover of $q$, for short). A motivation for this definition is given by the following theorem.

Theorem 4.8. The subspace $\bar{S}$ of $V^{\bar{S}}$ defines a quotient $\left(q_{E}^{\bar{S}, \bar{T}}\right)_{\bar{S}}$ of $q_{E}^{\bar{S}, \bar{T}}$. With an obvious identification of $V^{\bar{S}} / \bar{S}$ with $V$, we have $\left(q_{E}^{\bar{S}} \bar{T}\right)_{\bar{S}}=q$.

The proof of Theorem 4.8 is straightforward. We leave it to the reader. By this theorem and Theorem 4.6 we immediately obtain the following:

Corollary 4.9. The polar space associated to $q_{E}^{\bar{S}, \bar{T}}$ in $\mathrm{PG}\left(V^{\bar{S}}\right)$ is isomorphic to the polar space $S_{q}$ associated to $q$.

For $\bar{r} \in \bar{R}$, let $\theta(\bar{r})$ be the projection of $\bar{r}$ onto $\bar{S}$ along $\bar{T}$, namely $\theta(\bar{r})$ is the unique element of $\bar{S} \cap(\bar{r}+\bar{T})$. For every $q$-singular vector $x \in V$, the subspace $\langle x, \bar{S}\rangle$ of $V^{\bar{S}}$ contains a unique $q_{E}^{\bar{S}, \bar{T}}$-singular point, represented by the vector $x-\theta\left(\overline{g_{E}(x, x)}\right)$. Put

$$
e_{q, E}^{\bar{S}, \bar{T}}([x]):=\left[x-\theta\left(\overline{g_{E}(x, x)}\right)\right] .
$$

We can now rephrase Theorem 4.8 as follows.

Theorem 4.10. The mapping $e_{q, E}^{\bar{S}, \bar{T}}$ is a projective embedding of $S_{q}$ in $\operatorname{PG}\left(V^{\bar{S}}\right)$. The image $e_{q, E}^{\bar{S}, \bar{T}}\left(S_{q}\right)$ of $S_{q}$ by $e_{q, E}^{\bar{S}, \bar{T}}$ is the polar space associated to $q_{E}^{\bar{S}, \bar{T}}$ in $\operatorname{PG}\left(V^{\bar{S}}\right)$. Moreover, if $\pi_{\bar{S}}$ is the projection of $V^{\bar{S}}$ onto $V^{\bar{S}} / \bar{S}$, then the canonical isomorphism from $V^{\bar{S}} / \bar{S}$ to $V$ yields an isomorphism from the composition $\pi_{\bar{S}} \cdot e_{q, E}^{\bar{S}, \bar{T}}$ to the inclusion embedding $e_{q}: S_{q} \rightarrow \mathrm{PG}(V)$.

We call $e_{q, E}^{\bar{S}, \bar{T}}$ the lifting of $e_{q}$ to $V^{\bar{S}}$ based at $E$.

Remark 4.11. We have assumed that $q$ is non-trivial since the very beginning of Section 4, however the previous construction can be repeated when $q$ is trivial. In that case we choose a sesquilinearization $f$ of $q$ and we define $q_{E}^{\bar{S}} \bar{T}$ with the help of $f$, as in the non-trivial case, but the form $q_{E}^{\bar{S}} \bar{T}$ now depends on the particular choice of $f$. The form $q_{E}^{\bar{S}, \bar{T}}$ is non-trivial provided that $\bar{S} \neq\{\overline{0}\}$. It is still true that $q$ is a quotient of $q_{E}^{\bar{S}}, \bar{T}$, but Corollary 4.9 must be rephrased as follows: the polar space associated to $q_{E}^{\bar{S}, \bar{T}}$ in $\operatorname{PG}\left(V^{\bar{S}}\right)$ is isomorphic to $S_{f}$ (compare Theorem 4.6(2)).

\subsubsection{Independence of $q_{E}^{\bar{S}, \bar{T}}$ from the choice of $E$}

Our definition of $q_{E}^{\bar{S}, \bar{T}}$ rests on the choice of a particular ordered $q$-singular basis $E$. In this subsection we shall prove that this choice is ultimately irrelevant: different choices lead to isomorphic forms. 
Given two $q$-singular bases $E$ and $E^{\prime}$, let $\delta_{E, E^{\prime}}$ be the difference-map of the pair $\left(E, E^{\prime}\right)$ (see Subsection 3.4). Recall that $\delta_{E, E^{\prime}}(x) \in \bar{R}_{E, E^{\prime}} \subseteq \bar{R}$, by Lemma 3.11. Hence $\theta\left(\delta_{E, E^{\prime}}(x)\right)$ is defined for every $x \in V$, where $\theta$ is the projection of $\bar{R}$ onto $\bar{S}$ along $\bar{T}$, as in (36). In view of the definition of $\delta_{E, E^{\prime}}$, the following holds for every vector $x \in V$ :

$$
x-\theta\left(\overline{g_{E^{\prime}}(x, x)}\right)=x-\theta\left(\overline{g_{E}(x, x)}\right)+\theta\left(\delta_{E, E^{\prime}}(x)\right) .
$$

Let $\Delta_{E, E^{\prime}}: V^{\bar{S}} \rightarrow V^{\bar{S}}$ be the mapping defined as follows, for $x \in V$ and $\bar{r} \in \bar{S}$ :

$$
\Delta_{E, E^{\prime}}(x+\bar{r})=x+\theta\left(\delta_{E, E^{\prime}}(x)\right)+\bar{r} .
$$

Theorem 4.12. The mapping $\Delta_{E, E^{\prime}}$ is linear and bijective, it fixes $\bar{S}$ elementwise and yields an isomorphism from $q_{E}^{\bar{S}, \bar{T}}$ to $q_{E^{\prime}}^{\bar{S}, \bar{T}}$. Explicitly,

$$
q_{E}^{\bar{S}, \bar{T}}(x+\bar{r})=q_{E^{\prime}}^{\bar{S}, \bar{T}}\left(\Delta_{E, E^{\prime}}(x+\bar{r})\right)
$$

for any $x \in V$ and $\bar{r} \in \bar{S}$. Consequently, $\Delta_{E, E^{\prime}}$ is an isomorphism of embeddings from the lifting $e_{q, E}^{\bar{S}, \bar{T}}$ of $e_{q}$ based at $E$ to the lifting $e_{q, E^{\prime}}^{\bar{S}, \bar{T}}$ of $e_{q}$ based at $E^{\prime}$.

Proof. By Lemma 3.11, $\delta_{E, E^{\prime}}$ is a linear mapping from $V$ to $\bar{R}_{E, E}$. Hence $\Delta_{E, E^{\prime}}$ is linear. Clearly, $\Delta_{E, E^{\prime}}$ fixes $\bar{S}$ elementwise. Moreover the composition of $\Delta_{E, E^{\prime}}$ with the projection of $V^{\bar{S}}$ onto $V$ along $\bar{S}$ induces the identity mapping on $V$. Therefore $\Delta_{E, E^{\prime}}$ is bijective. We have

$$
\begin{aligned}
q_{E}^{\bar{S}, \bar{T}}(x+\bar{r}) & =\overline{g_{E}(x, x)}+\bar{r}+\bar{T} \\
& =\overline{g_{E^{\prime}}(x, x)}+\left(\overline{g_{E}(x, x)}-\overline{g_{E^{\prime}}(x, x)}\right)+\bar{r}+\bar{T} \\
& =\overline{g_{E^{\prime}}(x, x)}+\delta_{E, E^{\prime}}(x)+\bar{r}+\bar{T} \\
& =\overline{g_{E^{\prime}}(x, x)}+\theta\left(\delta_{E, E^{\prime}}(x)\right)+\bar{r}+\bar{T}=q_{E^{\prime}}^{\bar{S}, \bar{T}}\left(\Delta_{E, E^{\prime}}(x+\bar{r})\right) .
\end{aligned}
$$

(Recall that $\delta_{E, E^{\prime}}(x)+\bar{T}=\theta\left(\delta_{E, E^{\prime}}(x)\right)+\bar{T}$, by the definition of $\theta$.) Equation (37) is proved. Exploiting (37), it is not difficult to prove that $\Delta_{E, E^{\prime}}$ is an isomorphism from $e_{q, E}^{\bar{S}, \bar{T}}$ to $e_{q, E^{\prime}}^{\bar{S}, \bar{T}}$.

Theorem 4.12 allows us to drop the index $E$ in our notation, thus writing $q^{\bar{S}, \bar{T}}$ and $e_{q}^{\bar{S}, \bar{T}}$ for $q_{E}^{\bar{S}, \bar{T}}$ and $e_{q, E}^{\bar{S}, \bar{T}}$ whenever the particular choice of the basis $E$ is irrelevant for what we are saying. Accordingly, we call $q^{\bar{S}, \bar{T}}$ and $e_{q}^{\bar{S}, \bar{T}}$ the cover of $q$ via $(\bar{S}, \bar{T})$ and the lifting of $e_{q}$ to $V^{\bar{S}}$ respectively, with no mention of $E$. 


\subsubsection{Dominant covers}

As $\bar{S} \oplus \bar{T}=\bar{R}$, we have $\bar{S}=\bar{R}$ if and only if $\bar{T}=\{\overline{0}\}$. When $\bar{T}=\{\overline{0}\}$ the form $q^{\bar{S}, \bar{T}}=q^{\bar{R},\{\overline{0}\}}$ is pseudo-quadratic with defect equal to $\operatorname{Rad}(f) \oplus \bar{R}$. Improper covers are allowed too. We get them by taking $\bar{S}=\{\overline{0}\}$ (whence $\bar{T}=\bar{R}$ ). Clearly, $q^{\{\overline{0}\}, \bar{R}}=q$.

We have not assumed that $\bar{R} \neq\{\overline{0}\}$. Indeed the construction of $q^{\bar{S}, \bar{T}}$ makes sense even if $\bar{R}=\{\overline{0}\}$. In this case $q$ is pseudo-quadratic and $\bar{S}=\bar{T}=\{\overline{0}\}$, hence $q^{\bar{S}, \bar{T}}=q$, namely $q$ does not admit any proper cover. Conversely, if $q$ does not admit any proper cover then $\bar{R}=\{\overline{0}\}$. We say that $q$ is dominant if it does not admit any proper cover. So, the form $q^{\bar{S}, \bar{T}}$ is dominant if and only if $\bar{T}=\{\overline{0}\}$. We call $\left.q^{\bar{R},\{\overline{0}}\right\}$ the dominant cover of $q$.

\subsubsection{Quotients versus covers}

According to Theorem 4.8, if $\tilde{q}: \widetilde{V} \rightarrow \bar{K} / \bar{T}$ is a cover of $q: V \rightarrow \bar{K} / \bar{R}$ then $q$ is a quotient of $\tilde{q}$. A converse of this statement also holds.

Theorem 4.13. Given a subspace $\bar{T}$ of $\bar{K}^{\circ}$ and a generalized $(\sigma, \varepsilon)$-quadratic form $\tilde{q}: \widetilde{V} \rightarrow \bar{K} / \bar{T}$, let $U$ be a subspace of $\widetilde{V}$ defining a quotient of $\tilde{e}$. Then $\tilde{q}$ is isomorphic to a cover of the quotient $\tilde{q}_{U}$ of $\tilde{q}$ by $U$.

Proof. Put $V:=\widetilde{V} / U$ and $q:=\tilde{q}_{U}: V \rightarrow \bar{K} / \bar{R}$, where $\bar{R}:=\bar{T}_{U}$ is the subspace of $\bar{K}^{\circ}$ such that $\bar{R} / \bar{T}=\tilde{q}(U)$ (see Subsection 4.1). Let $\bar{S}$ be a complement of $\bar{T}$ in the $K$-vector space $\bar{R}$ and $W$ a complement of $U$ in $\widetilde{V}$. Let $\tilde{\pi}_{U}$ be the projection of $\widetilde{V}$ onto $V=\widetilde{V} / U$ and $\theta$ the projection of $\bar{R}$ onto $\bar{S}$ along $\bar{T}$. Let $\alpha: \widetilde{V} \rightarrow V^{\bar{S}}=V \oplus \bar{S}$ be the linear mapping defined by the following clauses: $\alpha(w)=\tilde{\pi}_{U}(w)$ for every $w \in W$ and $\alpha(u)=\theta(\tilde{q}(u))$ for every $u \in U$. As the reader can check, $\alpha$ is an isomorphism from $\tilde{q}$ to $q^{\bar{S}}, \bar{T}$.

Corollary 4.14. Let $q: V \rightarrow \bar{K} / \bar{R}$ be a non-trivial generalized $(\sigma, \varepsilon)$-quadratic form. Given a vector subspace $\bar{T}$ of $\bar{R}$, let $\bar{S}$ and $\bar{S}^{\prime}$ be two complements of $\bar{T}$ in $\bar{R}$. Then $q^{\bar{S}, \bar{T}} \cong q^{\bar{S}^{\prime}, \bar{T}}$.

Proof. The conclusion follows from the proof of Theorem 4.13 with $V^{\bar{S}^{\prime}}, q^{\bar{S}^{\prime}, \bar{T}}$ and $\bar{S}^{\prime}$ in the roles of $\widetilde{V}$, $\tilde{q}$ and $U$ respectively, recalling that, by Theorem 4.8, $q$ is the quotient of $q^{\prime}, \bar{T}$ over $\bar{S}^{\prime}$.

\subsubsection{Partial independence of $q^{\bar{S}, \bar{T}}$ from the choice of $\bar{S}$ and $\bar{T}$}

In general, if $\bar{R}=\bar{S} \oplus \bar{T}$ and $\bar{R}=\bar{S}^{\prime} \oplus \bar{T}^{\prime}$ are two decompositions of $\bar{R}$ then $q^{\bar{S}, \bar{T}} ¥ q^{\bar{S}^{\prime}, \bar{T}^{\prime}}$. However, with a suitable choice of $\bar{T}^{\prime}$ the forms $q^{\bar{S}, \bar{T}}$ and $q^{\bar{S}^{\prime}, \bar{T}^{\prime}}$ 
are weakly isomorphic in the sense of Subsection 3.5. Explicitly:

Proposition 4.15. With $\bar{S}, \bar{T}, \bar{S}^{\prime}$ and $\bar{T}^{\prime}$ as above, suppose that $K$ admits an automorphism $\rho$ stabilizing $(\sigma, \varepsilon)$ and such that the automorphism $\bar{\rho}$ of $\bar{K}$ induced by $\rho$ stabilizes $\bar{R}$ and maps $\bar{T}$ onto $\bar{T}^{\prime}$. Then the forms $q^{\bar{S}} \bar{T}$ and $q^{\bar{S}^{\prime}, \bar{T}^{\prime}}$ are weakly isomorphic.

Proof. Given a $q$-singular basis $E$ of $V$ let $\rho_{E}$ be the $\rho$-semi-linear mapping of $V$ that fixes all vectors of $E$ and, for $x \in V$ and $\bar{r} \in \bar{S}$, set $\rho_{E}(x+\bar{r}):=$ $\rho_{E}(x)+\bar{r}^{\bar{\rho}}$. Then $\rho_{E}$ is a bijective $\rho$-semilinear mapping from $V^{\bar{S}}$ to $V^{\bar{S}^{\bar{\rho}}}$ and $\left(q_{E}^{\bar{S}, \bar{T}}(x+\bar{r})\right)^{\bar{\rho}}=q_{E}^{\bar{S}^{\bar{\rho}}, \bar{T}^{\bar{\rho}}}\left(\rho_{E}(x+\bar{r})\right)$ for every vector $x+\bar{r}$ of $V^{\bar{S}}$. Hence $q^{\bar{S}, \bar{T}}$

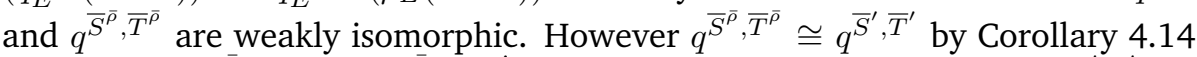
and because $\bar{R}^{\bar{\rho}}=\bar{R}$ and $\bar{T}^{\bar{\rho}}=\bar{T}^{\prime}$ by assumption. Therefore $q^{\bar{S}, \bar{T}}$ and $q^{\bar{S}^{\prime}, \bar{T}^{\prime}}$ are weakly isomorphic.

\section{Forms for embedded polar spaces}

Throughout this section $S=(P, L)$ is a non-degenerate polar space of rank at least 2 and $e: S \rightarrow \mathrm{PG}(V)$ is a projective embedding. So, the image $e(S)=$ $(e(P), e(L))$ of $S$ by $e$ is a full subgeometry of $\mathrm{PG}(V)$, it spans $\mathrm{PG}(V)$ and it is isomorphic to $S$.

Let $K$ be the underlying divison ring of $V$. By Theorem 1.1, an admissible pair $(\sigma, \varepsilon)$ of $K$ and a $(\sigma, \varepsilon)$-sesquilinear form $f: V \times V \rightarrow K$ exist such that $e(S)$ is a subspace of the polar space $S_{f}=\left(P_{f}, L_{f}\right)$ associated to $f$. Explicitly,

(E1) $e(P) \subseteq P_{f}$ and, for any two points $[x],[y] \in e(P)$, the line $[x, y]$ of $\mathrm{PG}(V)$ belongs $e(L)$ if and only if $f(x, y)=0$.

Property (E1) implies both the following:

(E2) For any two points $[x]$ and $[y]$ of $\mathrm{PG}(V)$ with $[y] \in e(P)$, we have $f(x, y)=$ 0 if and only if either the line $[x, y]$ belongs to $e(L)$ or $[x, y] \cap e(P)=\{[y]\}$.

(E3) $e(P) \cap[\operatorname{Rad}(f)]=\emptyset$.

As for (E3), recall that $S$ is non-degenerate by assumption while $f$ might be degenerate. By (E1), (E2) and (E3) and recalling that $e(P)$ spans $\mathrm{PG}(V)$, we also obtain the following:

(E4) A point $[x]$ of $\mathrm{PG}(V)$ belongs to $[\operatorname{Rad}(f)]$ if and only if every line of $\mathrm{PG}(V)$ through $[x]$ meets $e(P)$ in at most one point. 
The form $f$ is uniquely determined up to proportionality (Proposition 2.6). Moreover $f$ is trace-valued by Proposition 2.5, since $P_{f} \supseteq e(P)$ and $e(P)$ spans $\mathrm{PG}(V)$. Let $E=\left(e_{i}\right)_{i \in I}$ be a basis of $V$ such that $\left[e_{i}\right] \in e(P)$ for any $i \in I$. Such a basis exists since $e(P)$ spans $\mathrm{PG}(V)$. We call $E$ an $e(S)$-basis of $V$. Given a total ordering $<$ on $I$, let $g_{E}(x, y)$ be defined as in (25) of Subsection 3.4 and put

$$
\gamma_{E}(x):=\overline{g_{E}(x, x)}=\sum_{i<j} \overline{\lambda_{i}^{\sigma} f\left(e_{i}, e_{j}\right) \lambda_{j}} \text { for every vector } x=\sum_{i} e_{i} \lambda_{i} \in V .
$$

Lemma 5.1. The mapping $\gamma_{E}$ is a (possibly trivial) $(\sigma, \varepsilon)$-quadratic form, $g_{E}$ is a facilitating form for $\gamma_{E}$ and $f$ is a sesquilinearization of $\gamma_{E}$. The form $\gamma_{E}$ is trivial if and only if $\sigma=\mathrm{id}_{K}, \varepsilon=-1$ and $\operatorname{char}(K) \neq 2$.

Proof. The first three claims of this lemma are obvious. The last one follows from (4) of Subsection 2.1.

Let $\bar{R}$ be the closed subgroup of $\bar{K}$ generated by the set $\left\{\gamma_{E}(x)\right\}_{[x] \in e(P)}$ and define a mapping $q: V \rightarrow \bar{K} / \bar{R}$ as follows:

$$
q(x):=\gamma_{E}(x)+\bar{R} .
$$

Lemma 5.2. The mapping $q$ defined in (38) is a (possibly trivial) generalized $(\sigma, \varepsilon)$-quadratic form. If $q$ is non-trivial then $f$ is the sequilinearization of $q$. In this case $e(S)$ is a subspace of the polar space $S_{q}=\left(P_{q}, L_{q}\right)$ associated to $q$.

Proof. The first two claims of this lemma are straightforward. As for the third one, note firstly that $S_{q}$ is a subspace of $S_{f}$ since $f$ is the sesquilinearization of $q$. Clearly, $e(P) \subseteq P_{q}$. Therefore $e(S)$ is a subspace of $S_{q}$, as both $e(S)$ and $S_{q}$ are subspaces of $S_{f}$.

Lemma 5.2 and Corollary 3.4 imply the following:

Corollary 5.3. If $(\sigma, \varepsilon)$ is of trace type then either $\bar{R}=\bar{K}$ or $\bar{R}=\{\overline{0}\}$.

Note that, while $\gamma_{E}$ depends on the choice of the ordered basis $E$, neither $\bar{R}$ nor $q$ depend on that choice (see the final remark of Subsection 3.4).

Corollary 5.4. The form $q$ is trivial if and only if $\bar{R}=\bar{K}$. If $\gamma_{E}$ is trivial then $\bar{R}=\bar{K}$ (whence $q$ is also trivial)

Proof. The form $f$ is non-trivial, since $e(S)$ is a subspace of $S_{f}$ and it is nondegenerate. This fact and Proposition 3.5 imply the first claim of the corollary. According to the last claim of Lemma 5.1, the form $\gamma_{E}$ is trivial if and only if $f$ is alternating and $\operatorname{char}(K) \neq 2$. If this is the case then $\bar{R}=\bar{K}$. 
Theorem 5.5. Either $q$ is trivial or $e(S)=S_{q}$.

Proof. Suppose that $q$ is non-trivial. By Corollary 5.4, $\bar{R}$ is a proper subgroup of $\bar{K}$. Moreover $e(S)$ is a subspace of $S_{q}$, by the last claim of Lemma 5.2.

Let $\tilde{q}:=q^{\bar{R},\{\overline{0}\}}$ be the dominant cover of $q$ based at $E$, let $\tilde{f}:=f^{\bar{R}}$ be its sesquilinearization and put $\widetilde{V}:=V \oplus \bar{R}$. The embedding $e: S \rightarrow \mathrm{PG}(V)$ lifts to an embedding $\tilde{e}: S \rightarrow \mathrm{PG}(\widetilde{V})$, obtained as the composition of $e$ with the lifting of the inclusion embedding $e_{q}: S_{q} \rightarrow \mathrm{PG}(V)$ to $\widetilde{V}$ (see definition (36) of Subsection 4.2). Let $\widehat{V}$ be the subspace of $\widetilde{V}$ spanned by $\tilde{e}(P)$. We shall prove that $\widehat{V}=\widetilde{V}$.

Put $\widehat{R}:=\bar{R} \cap \widehat{V}$ and let $\hat{q}$ and $\hat{f}$ be the forms induced by $\tilde{q}$ and $\tilde{f}$ on $\widehat{V}$. Clearly, all points of $\tilde{e}(P)$ are $\hat{q}$-singular. As $\widehat{V}+\bar{R}=\widetilde{V}$, we have $\widehat{V} / \widehat{R} \cong \widetilde{V} / \bar{R} \cong V$ and $\widehat{R}$ defines a quotient $\hat{q}_{\widehat{R}}$ of $\hat{q}$. Via an obvious identification of $V$ with $\widehat{V} / \widehat{R}$, we may assume that $\hat{q}_{\widehat{R}}$ is defined over $V$. Accordingly, all points of $e(P)$ are $\hat{q}_{\widehat{R}}$-singular. It follows that $\gamma_{E}(x)$ belongs to the co-defect $\widehat{R}$ of $\hat{q}_{\widehat{R}}$, for every point $[x] \in e(P)$. However, $\bar{R}$ is generated by $\{\gamma(x)\}_{[x] \in e(P)}$. Therefore $\widehat{R}=\bar{R}$. Hence $\bar{R} \subseteq \widehat{V}$. It is now clear that $\widehat{V}=\widetilde{V}$, namely $\tilde{e}(P)$ spans $\operatorname{PG}(\widetilde{V})$.

Since $e(S)$ is a subspace of $S_{q}$, the image $\tilde{e}(S)$ of $S$ by $\tilde{e}$ is a subspace of the polar space $S_{\tilde{q}}=\left(P_{\tilde{q}}, L_{\tilde{q}}\right)$ associated to $\tilde{q}$. The latter is a subspace of the polar space $S_{\tilde{f}}=\left(P_{\tilde{f}}, L_{\tilde{f}}\right)$ associated to $\tilde{f}$. Hence $\tilde{e}(S)$ is also a subspace of $S_{\tilde{f}}$, namely $\tilde{e}(S)$ and $\tilde{f}$ satisfy (E1), whence (E2), (E3) and (E4) too.

We shall now prove that $e(S)=S_{q}$. Suppose the contrary, namely $e(P) \subset$ $P_{q}$. Then we also have $\tilde{e}(P) \subset P_{\tilde{q}}$. Let $[a] \in P_{\tilde{q}}-\tilde{e}(P)$. Suppose firstly that $[a] \notin[\operatorname{Rad}(\tilde{f})]$. By (E4), there exist two distinct points $[b],[c] \in \tilde{e}(P)$ such that the line $[b, c]$ contains $[a]$. We have $\tilde{f}(a, a)=\tilde{f}(b, b)=\tilde{f}(c, c)=0$ since all of $[a],[b]$ and $[c]$ belong to $P_{\tilde{f}}$. On the other hand, the line $[b, c]$ does not belong to $\tilde{e}(L)$, since it contains $[a]$ which, by assumption, does not belong to $\tilde{e}(P)$. Then $\tilde{f}(b, c) \neq 0$ by (E1). Since $\tilde{f}(b, b)=\tilde{f}(c, c)=0$ while $\tilde{f}(b, c) \neq 0$, the form $\tilde{f}$ induces a non-degenerate form on the subspace $\langle b, c\rangle$ of $\widetilde{V}$. Thus we can apply Proposition 10.3.10 of Buekenhout and Cohen [2]. By claim $(i)$ of that proposition, $P_{\tilde{q}} \cap[b, c]$ is the smallest subset of $S_{\tilde{f}} \cap[b, c]$ containing $[b]$ and $[c]$ and perspective with respect to the polarity $\delta_{\tilde{f},[b, c]}$ defined by $\tilde{f}$ on the line $[b, c]$. (We refer the reader to [2, Section 10.3] for the definition of sets perspective with respect to a polarity in a projective line.) However, $[b],[c] \in$ $\tilde{e}(P) \cap[b, c] \subseteq P_{\tilde{q}} \cap[b, c]$ and $\tilde{e}(P) \cap[b, c]$ is also perspective with respect to $\delta_{\tilde{f},[b, c]}$ by Proposition 10.3.4 of [2]. Hence $\tilde{e}(P) \cap[b, c]=P_{\tilde{q}} \cap[b, c]$. In particular, $[a] \in \tilde{e}(P)$, contrary to our choice of $[a]$. Therefore $[a] \in[\operatorname{Rad}(\tilde{f})]$, namely $[a] \in[\operatorname{Rad}(\tilde{q})]$, as $[a] \in P_{\tilde{q}}$. It follows that $P_{\tilde{q}}-\tilde{e}(P) \subseteq[\operatorname{Rad}(\tilde{q})]$.

Still with $[a] \in P_{\tilde{q}}-\tilde{e}(P) \subseteq[\operatorname{Rad}(\tilde{q})]$, let $[b] \in \tilde{e}(P)$. As both $[b]$ and $[a]$ are 
$\tilde{q}$-singular and $[a] \in[\operatorname{Rad}(\tilde{q})]$, the line $[a, b]$ belongs to $L_{\tilde{q}}$. Hence it is totally $\tilde{f}$-isotropic. By (E1), if $[a, b]$ contains a point of $\tilde{e}(P)$ different from $[b]$ then it also belongs to $\tilde{e}(L)$, but this contradicts the choice of $[a] \in P_{\tilde{q}}-\tilde{e}(P)$. Therefore $[a, b] \cap \tilde{e}(P)=\{[b]\}$, namely $[a, b]-\{[b]\} \subseteq P_{\tilde{q}}-\tilde{e}(P)$. However $P_{\tilde{q}}-\tilde{e}(P) \subseteq$ $[\operatorname{Rad}(\tilde{q})]$ and the latter is a subspace of $\operatorname{PG}(\widetilde{V})$. It follows that $[a, b] \subseteq[\operatorname{Rad}(\tilde{q})]$. This forces $[b] \in[\operatorname{Rad}(\tilde{q})] \cap \tilde{e}(P) \subseteq[\operatorname{Rad}(\tilde{f})] \cap \tilde{e}(P)$, a contradiction with (E3). We have reached a final contradiction. Therefore $e(S)=S_{q}$.

Let $\bar{R} \neq \bar{K}$. Then both $q$ and $\gamma_{E}$ are non-trivial (Corollary 5.4). Let $S_{\gamma_{E}}=$ $\left(P_{\gamma_{E}}, L_{\gamma_{E}}\right)$ be the polar space associated to $\gamma_{E}$ in $\mathrm{PG}(V)$. Clearly, $S_{\gamma_{E}}$ is a subspace of $S_{f}$.

Corollary 5.6. Let $\bar{R} \neq \bar{K}$. Then $S_{\gamma_{E}}$ is a subspace of $e(S)$. If moreover $(\sigma, \varepsilon)$ is of trace type then $S_{\gamma_{E}}=e(S)=S_{f}$.

Proof. The polar space $S_{\gamma_{E}}$ is a subgeometry of $S_{q}$. Moreover both $S_{\gamma_{E}}$ and $S_{q}$ are subspaces of $S_{f}$. Hence $S_{\gamma_{E}}$ is a subspace of $S_{q}$. However $S_{q}=e(S)$ by Theorem 5.5. Therefore $S_{\gamma_{E}}$ is a subspace of $e(S)$. Let $(\sigma, \varepsilon)$ be of trace type. Then $S_{\gamma_{E}}=S_{f}$ by Proposition 2.13. Hence $S_{\gamma_{E}}=e(S)=S_{f}$, since $S_{\gamma_{E}}$ is a subspace of $e(S)=S_{q}$ which in its turn is a subspace of $S_{f}$.

Remark 5.7. When $(\sigma, \varepsilon)$ is not of trace type it can happen that $S_{\gamma_{E}} \subset e(S)$. If that is the case then $S_{\gamma_{E}}$ depends on the choice of the basis $E$.

Theorem 5.8. Let $\bar{R}=\bar{K}$. Then $f$ is an alternating form and $e(S)=S_{f}$.

Proof. As $\bar{R}=\bar{K}$, the group $\bar{K}$ is generated by the elements $\gamma_{E}(x)$ for $[x] \in$ $e(S)$. However $e(S)$ is a subspace of $S_{f}$. Hence $\bar{K}$ is also generated by the elements $\gamma_{E}(x)$ for $x \in V$ such that $f(x, x)=0$. Given $x=\sum_{i} e_{i} \lambda_{i}$, let $t:=$ $\sum_{i<j} \lambda_{i}^{\sigma} f\left(e_{i}, e_{j}\right) \lambda_{j}$. Then

$$
f(x, x)=\sum_{i, j} \lambda_{i}^{\sigma} f\left(e_{i}, e_{j}\right) \lambda_{j}=\sum_{i \neq j} \lambda_{i}^{\sigma} f\left(e_{i}, e_{j}\right) \lambda_{j}+\sum_{i} \lambda_{i}^{\sigma} f\left(e_{i}, e_{i}\right) \lambda_{i} .
$$

However $f\left(e_{i}, e_{i}\right)=0$ for every $i \in I$ because $\left[e_{i}\right] \in e(P) \subseteq P_{f}$. Therefore

$$
\begin{aligned}
0 & =\sum_{i \neq j} \lambda_{i}^{\sigma} f\left(e_{i}, e_{j}\right) \lambda_{j}=\sum_{i<j} \lambda_{i}^{\sigma} f\left(e_{i}, e_{j}\right) \lambda_{j}+\sum_{i>j} \lambda_{i}^{\sigma} f\left(e_{i}, e_{j}\right) \lambda_{j} \\
& =\sum_{i<j} \lambda_{i}^{\sigma} f\left(e_{i}, e_{j}\right) \lambda_{j}+\sum_{j>i} \lambda_{j}^{\sigma} f\left(e_{j}, e_{i}\right) \lambda_{i} \\
& =\sum_{i<j} \lambda_{i}^{\sigma} f\left(e_{i}, e_{j}\right) \lambda_{j}+\sum_{i<j}\left(\lambda_{i}^{\sigma} f\left(e_{i}, e_{j}\right) \lambda_{j}\right)^{\sigma} \varepsilon \\
& =\sum_{i<j} \lambda_{i}^{\sigma} f\left(e_{i}, e_{j}\right) \lambda_{j}+\left(\sum_{i<j} \lambda_{i}^{\sigma} f\left(e_{i}, e_{j}\right) \lambda_{j}\right)^{\sigma} \varepsilon=t+t^{\sigma} \varepsilon .
\end{aligned}
$$

Hence $f(x, x)=0$ if and only if $t=-t^{\sigma} \varepsilon$, namely $t \in K^{\sigma, \varepsilon}$. However $\bar{K}$ is generated by $\left\{\gamma_{E}(x) \mid f(x, x)=0\right\}$. Therefore $K=K^{\sigma, \varepsilon}$. The latter holds 
precisely when $\varepsilon=-1$ and $\sigma=\mathrm{id}_{K}$, by (3) of Subsection 2.1. So, $\sigma=\mathrm{id}_{K}$ and $\varepsilon=-1$. In particular, $K$ is a field. If $\operatorname{char}(K) \neq 2$ then $f$ is alternating. Let $\operatorname{char}(K)=2$. Then $f$ is a symmetric bilinear form. However, $f$ is also tracevalued and the alternating forms are the only trace-valued symmetric bilinear forms in characteristic 2 . Hence $f$ is alternating.

We still must prove that $e(S)=S_{f}$. This can be proved with the help of Theorem 1.2, but we shall do without it. Instead of Theorem 1.2, we shall exploit properties (E1)-(E4) and a few results from [2, Chapter 10] on perspectivities of projective lines.

We firstly assume that $\operatorname{char}(K) \neq 2$. By way of contradiction, suppose that $P_{f} \not \subset e(P)$ and let $[a] \in P_{f}-e(P)$. Assume that $[a] \notin[\operatorname{Rad}(f)]$. By (E4), there exists at least one line $l$ of $\operatorname{PG}(V)$ containing $[a]$ and intersecting $e(P)$ in at least two points. By Proposition 10.3.4 of Buekenhout and Cohen [2], the set $e(P) \cap l$ is perspective with respect to the polarity $\delta_{f, l}$ defined by $f$ on the line $l$. However, according to [2, Proposition 10.3.10(ii)], the line $l$ does not contain any proper subset of size at least 2 and perspective with respect to $\delta_{f, l}$. Therefore $l=e(P) \cap l$. This contradicts the choice of $[a] \notin e(P)$. We must conclude that $[a] \in[\operatorname{Rad}(f)]$. So, $P_{f}-e(P) \subseteq[\operatorname{Rad}(f)]$. With $[a] \in$ $P_{f}-e(P) \subseteq[\operatorname{Rad}(f)]$, let $[b] \in e(P)$. Then $[a, b] \cap e(P)=\{[b]\}$ by (E1). Consequently $[a, b]-\{[b]\} \subseteq[\operatorname{Rad}(f)]$. However $\operatorname{Rad}(f)$ is a subspace of $V$. Hence $[b] \in[\operatorname{Rad}(f)]$, in contradiction with (E3). Therefore $e(S)=S_{f}$.

Let now $\operatorname{char}(K)=2$. Then $K_{\sigma, \varepsilon}=0, K^{\sigma, \varepsilon}=K$ and $\bar{K}=\bar{K}^{\circ}=K$. In particular, the scalar multiplication $\circ$ is defined over $K$ and $t \circ \lambda=t \lambda^{2}$ for any $t, \lambda \in K$. The additive group of $K$ equipped with $\circ$ as the scalar multiplication is a $K$-vector space. In order to distinguish between this vector space and the field $K$ itself we denote the latter by the letter $K$, keeping the symbol $\bar{K}$ for the vector space $(K, \circ)$. Given an element $t \in K$, if we regard it as a vector of $\bar{K}$ then we write $\bar{t}$ rather than $t$. Put $\widetilde{V}:=V \oplus \bar{K}$. The set $W:=\left\{x+\overline{\gamma_{E}(x)}\right\}_{[x] \in e(P)}$ is a subset of $\widetilde{V}$ and contains $E$. However $E$ spans $V$, the latter being now regarded as a subspace of $\widetilde{V}$. Therefore $\langle W\rangle \supseteq V$. It follows that $\langle W\rangle$ also contains the set $\left\{\overline{\gamma_{E}(x)}\right\}_{[x] \in e(P)}$. The latter spans $\bar{R}$ and $\bar{R}=\bar{K}$, by assumption. Therefore $W$ spans $\widetilde{V}$. We now define a quadratic form $\tilde{q}$ and an alternating form $\tilde{f}$ on $\tilde{V}$, as follows:

$$
\begin{array}{ll}
\tilde{q}(x+\bar{t})=\gamma_{E}(x)+t & \text { for all } x \in V \text { and } \bar{t} \in \bar{K} . \\
\tilde{f}(x+\bar{t}, y+\bar{s})=f(x, y) & \text { for all } x, y \in V \text { and } \bar{t}, \bar{s} \in K .
\end{array}
$$

It is readily seen that $\tilde{q}$ is indeed a quadratic form and $\tilde{f}$ is its sesquilinearization. Note that $\operatorname{Rad}(f)=\bar{K}$ and $\bar{K}$ contains no $\tilde{q}$-singular point. Hence $\tilde{q}$ is nonsingular. Accordingly, the polar space $S_{\tilde{q}}=\left(P_{\tilde{q}}, L_{\tilde{q}}\right)$ associated to $\tilde{q}$ in $\mathrm{PG}(\widetilde{V})$ is 
non-degenerate. Moreover $S_{\tilde{q}}$ is a subspace of the polar space $S_{\tilde{f}}$ associated to $\tilde{f}$, as $\tilde{f}$ is the sesquilinearization of $\tilde{q}$.

For $x \in V$ and $\bar{t} \in \bar{K}$ we have $\tilde{q}(x+\bar{t})=0$ if and only if $t=\gamma_{E}(x)$. Hence the set $\widetilde{P}:=\{[v]\}_{v \in W}$ is contained in $P_{\tilde{q}}$. It is not difficult to see that $\widetilde{P}$ is a subspace of $S_{\tilde{q}}$. Let $\widetilde{S}$ be the polar space induced by $S_{\tilde{q}}$ on $\widetilde{P}$. Clearly, $\widetilde{S}$ is a subspace of $S_{\tilde{q}}$. Hence it is also a subspace of $S_{\tilde{f}}$, since $S_{\tilde{q}}$ is a subspace of $S_{\tilde{f}}$. Since $\widetilde{P}$ spans $\widetilde{V}$ and $\widetilde{S}$ is a subspace of $S_{\tilde{q}}$, the radical of $\widetilde{S}$ is contained in the radical of $S_{\tilde{q}}$. However $S_{\tilde{q}}$ is non-degenerate. Hence $\widetilde{S}$ is non-degenerate. Consequently, property (E1), whence (E2), (E3) and (E4) hold for $\widetilde{S}$ and $\tilde{f}$.

We shall prove that $\widetilde{S}=S_{\tilde{q}}$. By way of contradiction, let $[a] \in P_{\tilde{q}}-\widetilde{P}$. Note that $a \notin \operatorname{Rad}(\tilde{f})$, because $S_{\tilde{q}}$ is non-degenerate. Then, by (E4) applied to $\widetilde{S}$ and $\tilde{f}$, there is a line $l$ of $\mathrm{PG}(\widetilde{V})$ containing $[a]$ and two distinct points $[b],[c] \in \widetilde{P}$. The line $l$ belongs to $L_{\tilde{q}}$, since it contains at least three distinct points of $P_{\tilde{q}}$ and $\tilde{q}$ is quadratic. Consequently, $l$ is totally singular for $\tilde{q}$. Hence $l$ is also totally isotropic for $\tilde{f}$. In particular $f(b, c)=0$. This forces $l$ to be a line of $\widetilde{S}$ too, a contradiction with the choice of $[a] \notin \widetilde{P}$. Therefore $\widetilde{S}=S_{\tilde{q}}$.

The projection $\pi_{\bar{K}}: \widetilde{V} \rightarrow \widetilde{V} / \bar{K}=V$ induces an isomorphism from $\widetilde{S}$ to $e(S)$. On the other hand, the quotient $\tilde{q}_{\bar{K}}$ of $\tilde{q}$ by $\bar{K}$ is trivial. Hence $\pi_{\bar{K}}$ induces an isomorphism from $S_{\tilde{q}}$ to $S_{f}$, by claim (2) of Theorem 4.6. However $S_{\tilde{q}}=\widetilde{S}$. Therefore $e(S)=S_{f}$.

\section{Initial embeddings}

In this section we shall revisit Theorem 1.2, giving an elementary proof of the fact that the embeddings considered in that theorem are dominant.

With $e: S \rightarrow \mathrm{PG}(V)$ and $f: V \times V \rightarrow K$ as in the previous section, let $q: V \rightarrow \bar{K} / \bar{R}$ be the generalized pseudo-quadratic form defined as in (38). By Theorems 5.5 and 5.8, either $q$ is non-trivial and $e(S)=S_{q}$ or $K$ is a field, $f$ is alternating and $e(S)=S_{f}$. The existence of the cover $q^{\bar{R},\{\overline{0}\}}$ makes it clear that, if $e(S)=S_{q}$, then $e$ is dominant only if $\bar{R}=\{\overline{0}\}$, namely $q$ is pseudo-quadratic. Conversely,

Lemma 6.1. Suppose that either $q$ is pseudo-quadratic or $f$ is alternating and $\operatorname{char}(K) \neq 2$. Then $e$ is dominant.

Proof. This lemma is contained in Theorem 1.2 but, since we are revisiting Theorem 1.2, we shall give a proof independent of that theorem.

Let $\tilde{e}: S \rightarrow \operatorname{PG}(\widetilde{V})$ be the hull of $e$. Then there exists a reflexive sesquilinear form $\tilde{f}: \widetilde{V} \times \widetilde{V} \rightarrow K$ such that $\tilde{e}(S)$ is a subspace of $S_{\tilde{f}}$. Let $\tilde{q}: \widetilde{V} \rightarrow \bar{K} / \bar{R}$ 
be the generalized pseudo-quadratic form defined as in (38) but with $V$ and $f$ replaced with $\widetilde{V}$ and $\tilde{f}$ respectively. By Theorems 5.5 and 5.8, either $\bar{R} \subset \bar{K}$ and $\tilde{e}(S)=S_{\tilde{q}}$ or $\bar{R}=\bar{K}$ and $\tilde{e}(S)=S_{\tilde{f}}$.

As $\tilde{e}$ is the hull of $e$, there exists a subspace $U$ of $\operatorname{Rad}(\tilde{f})$ such that $e \cong \tilde{e} / U$. If $\tilde{e}(S)=S_{\tilde{f}}$ then $\tilde{f}$ is non-degenerate. In this case $U=\{0\}$, whence $e \cong \tilde{e}$, namely $e$ is dominant. Suppose now that $\bar{R} \subset \bar{K}$. Then $\tilde{e}(S)=S_{\tilde{q}}$ and $e(S)=S_{\tilde{q}_{U}}$, where $\tilde{q}_{U}$ is the quotient of $\tilde{q}$ by $U$, regarded as a generalized pseudo-quadratic form on $V$ via an obvious identification of $V$ with $\widetilde{V} / U$. Then $q$ and $\tilde{q}_{U}$ are proportional, by Theorem 3.13. Hence $\tilde{q}_{U}$ is a pseudo-quadratic form. However pseudo-quadratic forms do not admit proper covers (Subsection 4.2.2), while $\tilde{q}$ is a cover of $\tilde{q}_{U}$ by Theorem 4.13. Hence $\tilde{q}_{U} \cong \tilde{q}$, namely $U=\{0\}$. Again, $e \cong \tilde{e}$.

Turning back to the general case, when $\bar{R} \subset \bar{K}$ we denote by $\tilde{e}$ the composition of $e$ with the lifting of $e_{q}: S_{q} \rightarrow \operatorname{PG}(V)$ to $\widetilde{V}:=V^{\bar{R}}$. Thus, $\tilde{e}(S)=S_{\tilde{q}}$, where $\tilde{q}:=q^{\bar{R},\{\overline{0}\}}$ is the dominant cover of $q$, as in the proof of Theorem 5.5. When $\bar{R}=\bar{K}$ and $\operatorname{char}(K) \neq 2$ we set $\widetilde{V}:=V$ and $\tilde{e}:=e$. Finally, let $\bar{R}=\bar{K}$ but $\operatorname{char}(K)=2$. It is well known that in this case $e$ is a quotient of an embedding $\tilde{e}: S \rightarrow \operatorname{PG}(\tilde{V})$, where $\tilde{e}(S)=S_{\tilde{q}}$ for a suitable quadratic form $\tilde{q}: \widetilde{V} \rightarrow K$ (see e.g. De Bruyn and Pasini [5]).

Theorem 6.2. In each of the cases considered above the embedding ẽ is dominant, whence it is the hull of $e$.

Proof. This statement immediately follows from Lemma 6.1, recalling that dominat covers of generalized pseudo-quadratic forms are pseudo-quadratic forms (Subsection 4.2.2).

Corollary 6.3. With ẽ as above, assume moreover that $S$ has rank at least 3 . Then ẽ is initial.

Proof. Embeddable polar spaces of rank at least 3 satisfy the conditions of the main theorem of Kasikova and Shult [8], which are sufficient for the existence of a $K$-initial embedding. Therefore the embedding $\tilde{e}$, being dominant, is also also $K$-initial (see Subsection 1.3.3). On the other hand, since $K$ coordinatizes the planes of $S$, all projective embeddings of $S$ are $K$-embeddings, namely $S$ is defined over $K$. Hence $\tilde{e}$ is initial.

The statement of Corollary 6.3 is included in Theorem 1.2, which is a rephrasing of Theorem 8.6 of Tits [10], but the proof given by Tits for that theorem is rather different from our proof of Corollary 6.3. In our proof we exploit the main result of Kasikova and Shult [8], which can be applied to polar spaces of 
rank at least 3 thanks to the fact that the maximal singular subspaces of such a polar space are projective spaces of dimension at least 2, while Tits's proof relies on a deep investigation of projective lines (see [10, 8.12]) and also applies to polar spaces of rank 2 , but for the two exceptional cases described in the following theorem (and mentioned in Theorem 1.2).

Theorem 6.4. [Tits $[10,8.6]]$ The embedding $\tilde{e}$ is initial even if $S$ has rank 2, except in the following two cases:

(1) $S$ is a grid and $|K|>4$.

(2) $K$ is a quaternion division ring, $\widetilde{V}=V(4, K)$ and, modulo proportionality and isomorphisms, $\varepsilon=-1, \sigma$ is the standard involution of $K$, we have $K_{\sigma, \varepsilon}=Z(K)$ and $\tilde{q}\left(x_{1}, x_{2}, x_{3}, x_{4}\right)=x_{1}^{\sigma} x_{2}+x_{3}^{\sigma} x_{4}+K_{\sigma, \varepsilon}$ for every vector $\left(x_{1}, x_{2}, x_{3}, x_{4}\right) \in \widetilde{V}$.

In case (1) we have as many isomorphism classes of projective embeddings as the cosets of $\mathrm{P} \Gamma \mathrm{L}(2, K)$ in the group of all permutations of the set $\mathrm{PG}(1, K)$. In case (2) only two isomorphism classes of projective embeddings exist. In either case, all projective embeddings of $S$ are dominant.

\section{References}

[1] A. Bak, On modules with quadratic forms, Algebraic K-Theory and its Geometric Applications (Conf., Hull, 1969), pp. 55-66, Springer, Berlin.

[2] F. Buekenhout and A. M. Cohen, Diagram Geometries, Springer, Berlin, 2013.

[3] F. Buekenhout and C. Lefèvre, Generalized quadrangles in projective spaces, Arch. Math. 25 (1974), 540-552.

[4] F. Buekenhout and E. E. Shult, On the foundations of polar geometry, Geom. Dedicata 3 (1974), 155-170.

[5] B. De Bruyn and A. Pasini, On symplectic polar spaces over non-perfect fields of characteristic 2, Linear Multilinear Algebra 47 (2009), 567-575.

[6] K. J. Dienst, Verallgemainerte Vierecke in projectiven Räumen, Arch. Math. 35 (1980), 177-186.

[7] A. Hahn and O. T. O'Meara, The classical groups and K-theory, with a foreword by J. Dieudonné, Grundlehren Math. Wiss. 291, SpringerVerlag, Berlin, 1989. 
[8] A. Kasikova and E. E. Shult, Absolute embeddings of point-line geometries, J. Algebra 238 (2001), 100-117.

[9] M. A. Ronan, Embeddings and hyperplanes of discrete geometries, $\mathrm{Eu}-$ ropean J. Combin. 8 (1987), 179-185.

[10] J. Tits, Buildings of Shperical Type and Finite BN-pairs, Springer Lecture Notes 386 (1974), Springer, Berlin.

[11] J. Tits and R. M. Weiss, Moufang Polygons, Springer, Berlin, 2002.

[12] F. D. Veldkamp, Polar Geometry I-V, Indag. Math. 21 (1959), 512-551 and 22 (1959), 207-212.

Antonio Pasini

Department of Information EngineERing and Maths., University of Siena, Via Roma 56, 53100 SiENA, ITALY

e-mail: antonio.pasini@unisi.it 